\title{
Fluorescent aptamers for detection and treatment of pathogenic bacteria and cancer
}

\author{
Aarushi Sharma ${ }^{1 *}$ and Grace Ramena ${ }^{2}$ \\ 1 Department of Pharmacology, Simmons Cancer Institute ${ }^{1}$, Southern Illinois School of Medicine, \\ Springfield, Illinois 62702, USA \\ 2 Department of Aquaculture and Fisheries, University of Arkansas, Pine Bluff, Arkansas 71601, USA \\ * Correspondence: ramenag@uapb.edu; Tel.: (870-575-8137)
}

\begin{abstract}
Issues presented by the application of monoclonal antibodies in diagnostic assays and as curative agents can make the use of such molecules costprohibitive and sometimes even unsafe. This has warranted the development of short single-stranded oligonucleotides known as Aptamers. The structural malleability of these short DNA or RNA nucleotide segments allows them to exist in distinct conformations. SELEX (Systematic Evolution of Ligands by Exponential Enrichment) is a multi-step process for synthesis of aptamers. Each step of this procedure is governed by a diverse set of factors that influence production efficiency, binding affinity, and specificity of the oligonucleotides. Headway in aptamer research has been made in recent years by the introduction of newer iterations of the SELEX process. A greater number of studies are now being carried out to incorporate aptamers into existing disease detection tools and therapies. An overview has been given first on the key aptamer properties and the process of their production (with its newer iterations), contrasting each of them with that of monoclonal antibodies. Possible manifold applications afforded due to unique aptamer characteristics are also discussed. A keen review is further provided on the design, development and use of fluorescent aptamers in bioimaging, sequencing or profiling, and treatment of pathogenic bacteria and tumor cells.
\end{abstract}

Keywords: aptamer; aptasensor; diagnosis; imaging; sequencing; therapeutics; probes; fluorescence; pathogenic bacteria; cancer cells; monoclonal antibodies; SELEX; nucleic acids.

\footnotetext{
${ }^{1}$ Doping is defined as the method of introducing a metal ion called dopant (lanthanide or actinide in the text example) into the already existing nanocrystal structure of another metal to enhance optical or electrochemical properties of the latter.

${ }^{2}$ Photo-blinking is defined as intermittent emission of fluorescent signals seen during continuous excitation of a fluorophore.
} 


\section{Introduction}

Interest in understanding the mechanics of cellular pathways and the need for specific targeting of therapeutics towards cancerous or infected cells provided the impetus to design short nucleotides known as Aptamers. Aptamer, a term derived from a combination of Latin and Greek words is apropos to and highlights the property of a synthetic single-stranded RNA or DNA derived oligonucleotide to form a tertiary structure that binds to its cognate cellular target with heightened affinity and specificity (Ellington \& Szostak, 1990; Zhou \& Rossi, 2017). The concept of aptamers was first born during the research to formulate effective treatments against virulent agents like HIV and adenoviruses (Sullenger, Gallardo, Ungers, \& Gilboa, 1990; Burgert, Ruzsics, Obermeier, Hilgendorf, Windheim, \& Elsing, 2002). The natural tendency of viral pathogens to synthesize nucleic acid-derived simple ligands binding host cell surface receptors was observed and that underpinned the development of such short fragments of nucleic acids.

Touted as "chemical antibodies" these nucleic acid fragments are potential contenders for Paul Ehrlich's famous "magic bullet" title (Zhou \& Rossi, 2017). However, both monoclonal antibodies and aptamers fall short of claiming such a name. Despite the introduction of human-like or humanized monoclonal antibodies to minimize antibody-induced immune reactions in recipients, most antibody treatments still struggle to strike the right balance between antigenicity and specificity (Hidding, 2016). Not to mention that antibodies have larger mass and it can be tedious to structurally alter them. Although aptamers mimic antibodies, they do not have similar drawbacks. Nonetheless, their design has been a very timeconsuming ordeal. After being ignored for almost three decades, there has been an impressive increase observed in the study, production, and utilization of aptamers for in-vitro and in-vivo diagnosis in recent years (Jayasena, 1999). Aptamers are now also being tested as promising therapeutics against various maladies (Zhou \& Rossi, 2017; Kaur, Bruno, Kumar, \& Sharma, 2018). Here, the structural nuances, properties, and the process of development of aptamers, contrasting each of them with those of monoclonal antibodies have been briefly introduced. Following that major applications of aptamers have been delineated with the ones used against pathogenic bacteria and tumors described in detail.

\footnotetext{
${ }^{1}$ Doping is defined as the method of introducing a metal ion called dopant (lanthanide or actinide in the text example) into the already existing nanocrystal structure of another metal to enhance optical or electrochemical properties of the latter.

${ }^{2}$ Photo-blinking is defined as intermittent emission of fluorescent signals seen during continuous excitation of a fluorophore.
} 


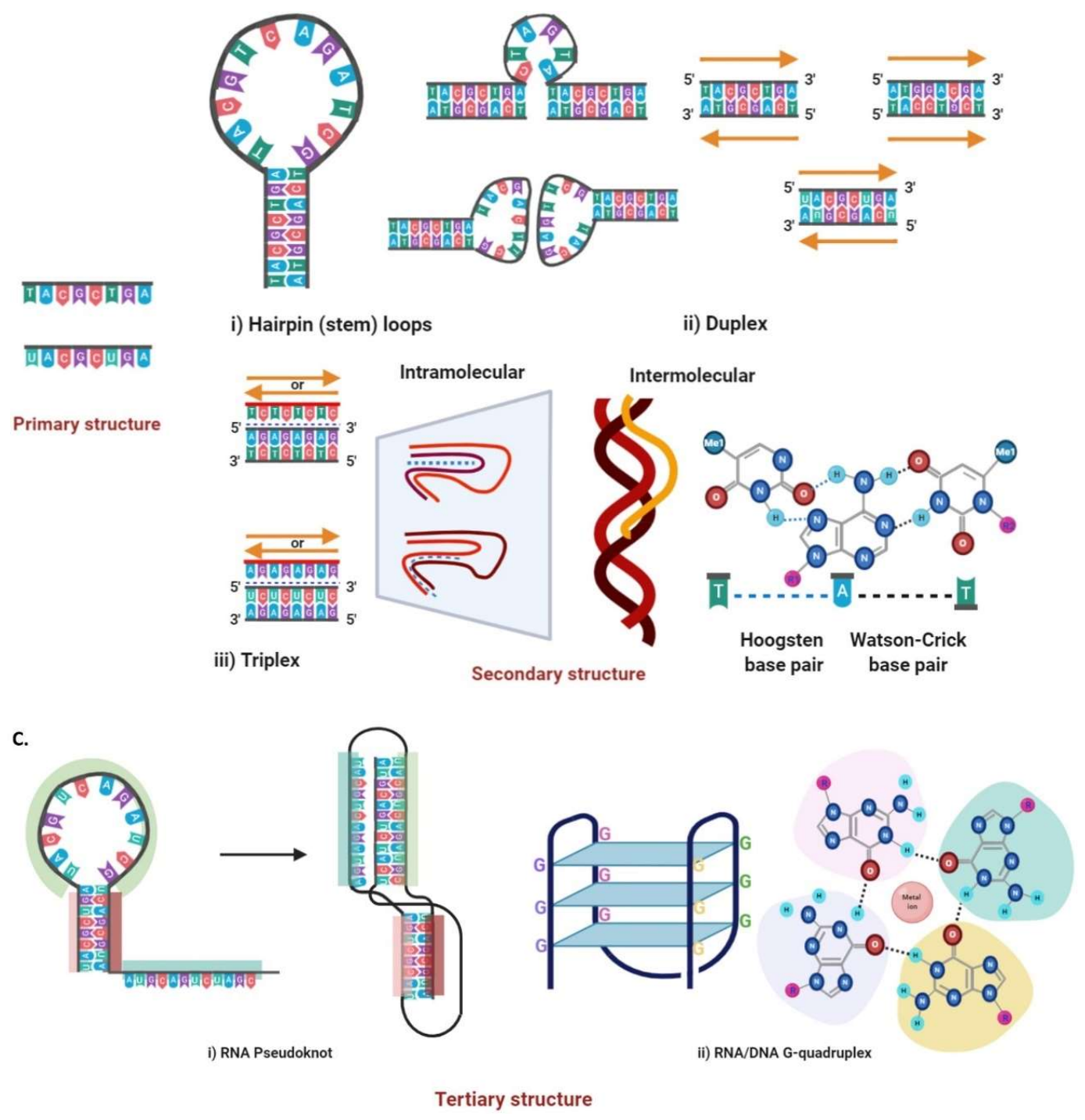

Figure 1.1 Different 2D/3D conformations of aptamers. RNA and DNA aptamers can exist as A. primary structure; B. secondary structure; or C. tertiary structure.

\footnotetext{
${ }^{1}$ Doping is defined as the method of introducing a metal ion called dopant (lanthanide or actinide in the text example) into the already existing nanocrystal structure of another metal to enhance optical or electrochemical properties of the latter.

${ }^{2}$ Photo-blinking is defined as intermittent emission of fluorescent signals seen during continuous excitation of a fluorophore.
} 
4 of 52

\section{Development of aptamers}

\subsection{Structure, types, and process of synthesis of aptamers}

Akin to proteins, non-covalent (electrostatic and non-electrostatic) interactions between nucleotides and their bases allow the formation of numerous secondary and tertiary structural patterns - hairpins and multi-loops, duplexes, triplexes, pseudoknots and guanine-rich G-quadruplexes (de-los-Santos-Álvarez et al., 2008; Zhang, Oni, \& Liu, 2017; Bing et al., 2017; Li, Syed, \& Sugiyama, 2016; Feigon, Dieckmann, \& Smith, 1996; Figure 1.1). Hairpin or stem-loop is a commonly observed secondary structure of RNA where A, U, G, C nucleotides form hydrogen bonds with complementary nucleotides on a wrinkled or folded back segment of the same strand (Varani, 1995; Svoboda \& Cara, 2006). The length of the stem (4-8 nucleotides), loop (6-7 nucleotides), nature and strength of interactions between the two segments of the strand forming stem (high GC content, less mismatch, base stacking, etc.) determine the thermodynamic stability of a hairpin. The three-leaved clover shape of tRNA is a prime example of RNA hairpins (Widmann et al., 2005). Nucleotide duplexes predominantly appear as a feature of DNA double helix although anti-parallel and parallel double-stranded RNA or DNA-RNA hybrid duplexes are naturally occurring too (Szabat \& Kierzek, 2017; Zhang et al., 2019). A triplex or triple helix is formed when shorter RNA or DNA oligonucleotides bind to a strand of DNA double helix which is either purine only or exhibits oligopurine rich portions largely in the major grooves (Roberts \& Crothers, 1992). Pseudoknots are one of the tertiary structural motifs adopted by nucleotides (Duca et al., 2008; Rietveld et al., 1982). As described in the study of its prototype discovered in a plant viral RNA, the simplest of these motifs are generated when hairpin loops form complementary associations with nucleotides outside the stem region but within the same strand (Duca et al., 2008; Rietveld et al., 1982; Peselis \& Serganov, 2014). Topologically there is no presence of a knot, but the hairpin loop pairings described above allow stacking of a hairpin stem into a tertiary form. G-quadruplexes are composed of four guanine rich strand segments stabilized by Hoogsteen base pairings (a variation of Watson-Crick base pairing) (Bing et al., 2017). From a planar view, quadruplexes appear as an arrangement popularly known as a G-quartet that is comprised of four guanines bound by eight Hoogsteen hydrogen bonds (Figure 1.1). Myriads of such folding features are adopted separately or in several

\footnotetext{
${ }^{1}$ Doping is defined as the method of introducing a metal ion called dopant (lanthanide or actinide in the text example) into the already existing nanocrystal structure of another metal to enhance optical or electrochemical properties of the latter.

${ }^{2}$ Photo-blinking is defined as intermittent emission of fluorescent signals seen during continuous excitation of a fluorophore.
} 
combinations by functional nucleic acid forms such as ribozymes, DNAzymes, tRNAs, ribosomal switches, and aptamers.

Aptamer cores are essentially a collection of 15 to 50 RNA or DNA nucleotides (target binding segment is 10-15nt long) arranged in a single strand that can exist in various folding states or conformations described above (Tantirungrotechai \& Soontornworajit, 2016; Chinnappan et al., 2020). The superior affinity and specificity of aptamers provides the ability to exist in several combinations of the folding structures placed together via interaction of hydrophobic, electrostatic, van der Waals, or hydrogen bonds (Song, Lee, \& Ban, 2012; Lodish et al., 2000). It was almost a decade after virus-protein interactions gave rise to the idea of developing an aptamer-like structure for therapy that aptamers were first synthesized (Ellington \& Szostak, 1990; Gold, 2015; Tuerk \& Gold, 1990; Robertson \& Joyce, 1990; Li, 2015). Three groups independently devised a technique to successfully isolate RNA aptamers in 1990. Amongst them, the most prominent work was by Dr. Larry Gold and his student Craig Tuerk at the University of Colorado. Their seminal study on aptamer synthesis created the SELEX process - Systematic Evolution of Ligands by Exponential Enrichment. Using SELEX, this duo isolated and enriched two highaffinity RNA ligands that bind to bacteriophage T4 DNA polymerase (Gold, 2015; Tuerk \& Gold, 1990; Robertson \& Joyce, 1990; Li, 2015; Zhuo et al., 2017). The first step in the general process of aptamer synthesis through SELEX involves assembling a single-stranded DNA or RNA library (Figure 1.2 A). The starting oligonucleotides are typically 20-60 nucleotides long (unlike 8 nucleotide length used by Tuerk and Gold) and have definitive primer binding structures at both ends of the unknown sequence region. This large and randomly selected pool of nucleotides $\left(\sim 10^{15}\right)$ is then incubated with several copies of the target molecule bound onto a carrier protein (Zhuo et al., 2017). After the unbound oligonucleotides are washed away, the solution containing target molecule-oligonucleotide complexes undergoes PCR amplification. The PCR products are purified to separate the complementary strands; this step prepares another library ready to experience the whole cycle of target binding, washing/filtration of unbound strands, and PCR amplification, repeatedly until a highly enriched cohort of target-specific oligonucleotides with optimal binding affinity is obtained.

Most SELEX processes require 5-15 cycles for attaining such an oligonucleotide pool; the selective library is further subjected to sequencing out of which a subset is

\footnotetext{
${ }^{1}$ Doping is defined as the method of introducing a metal ion called dopant (lanthanide or actinide in the text example) into the already existing nanocrystal structure of another metal to enhance optical or electrochemical properties of the latter.

${ }^{2}$ Photo-blinking is defined as intermittent emission of fluorescent signals seen during continuous excitation of a fluorophore.
} 
then chemically synthesized and assessed for structure, affinity, target specificity, and other physical properties. Ellington and Szostak from Mass. General Hospital and Gerald Joyce's group at Scripps Institute also demonstrated aptamer generation via the evolutionary selection simulating SELEX technique during 1990 (Ellington \& Szostak, 1990; Tuerk \& Gold, 1990; Robertson \& Joyce, 1990). Since then, SELEX has been modified into 25 or more variations (Zhuo et al., 2017; Aquino-Jarquin \& Toscano-Garibay, 2011; Darmostuk et al., 2015; Kushwaha et al., 2019; Blind \& Blank, 2015). RNA generating SELEX procedure requires a step of reverse transcription into cDNA before PCR amplification which, therefore, excludes the strand separation phase in the cycle. Depending on the target carrier or environment, there are three major adaptations of a SELEX method - protein-purified, whole-cell based, and live animal-based (Zhou \& Rossi, 2017). In the protein-purified kind, the target is bound to an artificial protein carrier. This allows flexibility to choose the mode of washing or filtering out the unbound oligonucleotides (Zhou \& Rossi, 2017; Nimjee, Rusconi, \& Sullenger, 2005). Nitrocellulose membrane was used as a filtration device for conventional SELEX. Other ways that have been implemented for this step include affinity or resin-matrix chromatography, magnetic beads, or chip-based purification. The use of protein-purified SELEX design encompasses minimal sophisticated instruments, ease of modifying experimental conditions for selection, and effective aptamer production for intracellular or extracellular proteins (Zhou \& Rossi, 2017). The whole-cell based variation allows target proteins to be in their natural environment and conformation while interacting with a given starter oligonucleotide library (Zhou \& Rossi, 2017; Shangguan et al., 2006; Sefah et al., 2010). The principle behind this SELEX variation is the difference between targetexpressing cells and non-expressing cells. Hence, the first step involves the introduction of a 'negative cell population' that leads to incubation of the unbound sequences with the 'target-positive cell population'. This procedure is popular for discovering ligands for protein targets with unknown structural properties. Removal of dead cells from both cell populations and simultaneous recovery of healthy cells are critical to ensure minimum loss of oligonucleotides in binding to dead cells. Live animal-based or genomic SELEX precludes the failure of in vitro produced aptamers from functioning inside a live organism (Zhou \& Rossi, 2017; Sola et al., 2020). It is accomplished by injecting the nucleotide library directly into the organ or tissue of interest in a live animal. The organ or tissue of interest is

\footnotetext{
${ }^{1}$ Doping is defined as the method of introducing a metal ion called dopant (lanthanide or actinide in the text example) into the already existing nanocrystal structure of another metal to enhance optical or electrochemical properties of the latter.

${ }^{2}$ Photo-blinking is defined as intermittent emission of fluorescent signals seen during continuous excitation of a fluorophore.
} 
harvested after a certain period of time has passed during which an appropriate amount of target protein-aptamer complexes may have formed; the bound aptamers are recovered from the tissue extract, purified, and then amplified before being sequenced.

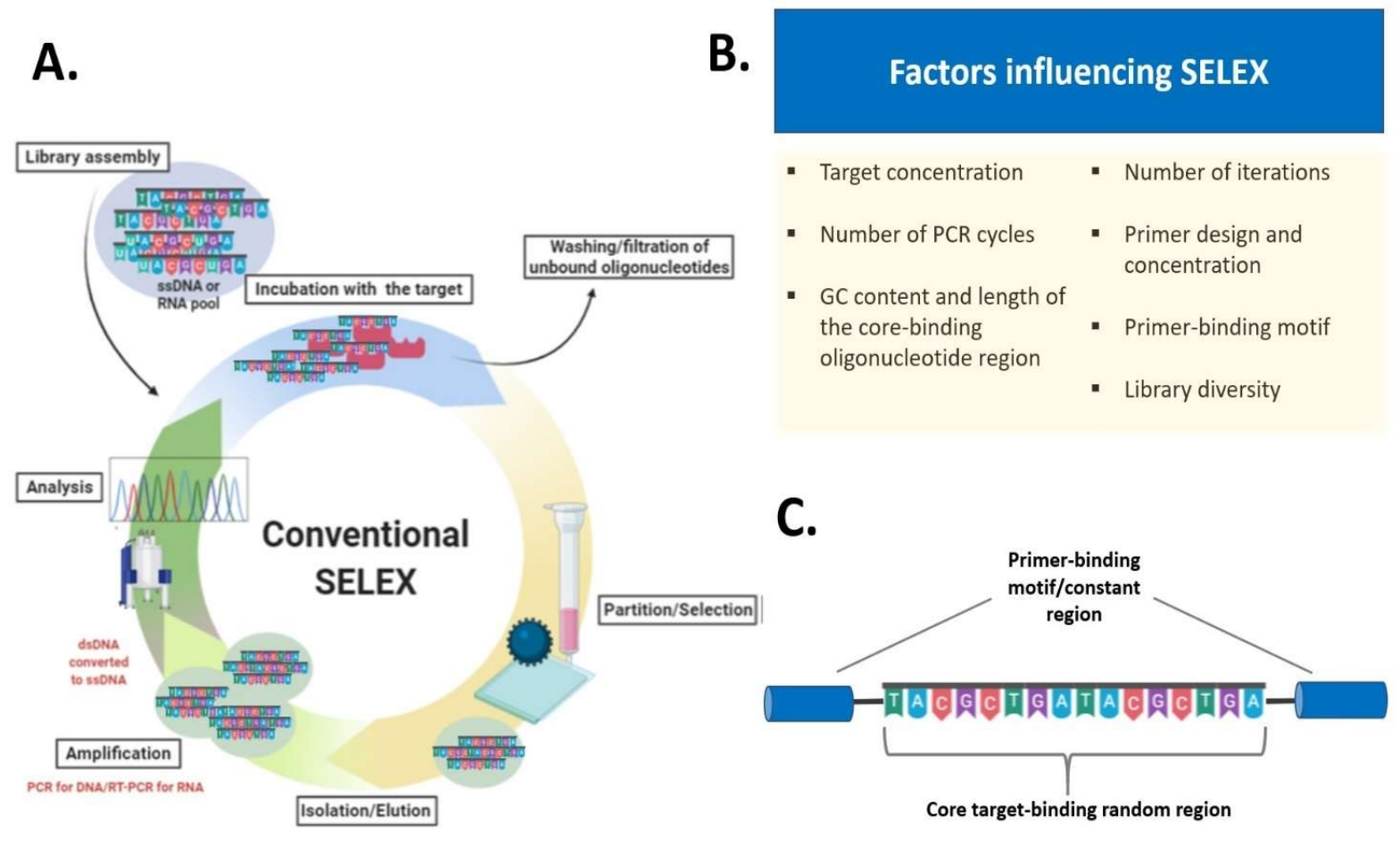

Figure 1.2 The various steps of a conventional SELEX process for the synthesis of aptamers and the factors it depends upon. A. SELEX can be divided into 7 basic stages; B. Some of the major factors that govern SELEX and consequently, aptamer design; C. structure of an RNA or DNA aptamer.

A successful execution of any SELEX process is contingent on several basic parameters (Figure 1.2 B). Library design for SELEX is a major limiting factor that is dependent upon quality and length of oligonucleotides, random region, fixed primer binding sequences, and overall structural stability of the aptamers (Komarova \& Kuznetsov, 2019; McKeague et al., 2015; Pobanz \& Lupták, 2016). Oligonucleotide quality relies, among other factors, on nucleotide ratio and distribution that determine the level of structural complexity/diversity of an aptamer library (Aquino-Jarquin \& Toscano-Garibay, 2011; Pan \& Clawson, 2009).

\footnotetext{
${ }^{1}$ Doping is defined as the method of introducing a metal ion called dopant (lanthanide or actinide in the text example) into the already existing nanocrystal structure of another metal to enhance optical or electrochemical properties of the latter.

${ }^{2}$ Photo-blinking is defined as intermittent emission of fluorescent signals seen during continuous excitation of a fluorophore.
} 
8 of 52

The random region forming the core of a starter oligonucleotide should have an optimal nucleotide number for adequate library diversity (Komarova \& Kuznetsov, 2019; McKeague et al., 2015; Pobanz \& Lupták, 2016; Figure 1.2 C). The greater the library diversity, the higher the chances for discovering target-binding sequence (Komarova \& Kuznetsov, 2019). Furthermore, long-length libraries are conducive for speedier selection and easier isolation steps; expansive random region allows for a higher probability of complex motifs to be present and has low functional interference with the primer sites. Regardless, data mining and analyses have found a weak correlation of nucleic acid type and aptamer length to the binding affinity (KD) (Thiel, Bair, Wyatt Thiel, Dassie, Rockey, Howell, Liu, Dupuy, Huang, Owczarzy, Behlke, McNamara, \& Giangrande,, 2011). In the same study though it was indicated that a strong correlation does exist between aptamer template length and target type. The core-flanking primer sites are ideally between 18 to 21 nucleotides long (Komarova \& Kuznetsov, 2019; Vorobyeva et al., 2018; Figure 1.2 C). Similar to the core, primer sites regulate aptamer structure, and perhaps, its complexity and stability (Wang et al., 2019). Formation of self-dimers or primerdimers can impede the amplification of the core region. The primer-binding region can also inhibit the interaction between the target and the binding motif or reduce the selection efficiency by decreasing library diversity (Komarova \& Kuznetsov, 2019; Vorobyeva et al., 2018; Pan \& Clawson, 2009). Some primer site sequences give rise to PCR by-products that have the potential to slow down the selection phase. These fixed aptamer end sequences when used for picking desired oligonucleotide ligands can also negatively alter their binding affinity because of being snipped off at the end of the selection and isolation phases. Upcoming studies have tried to prevent primer sites from negatively affecting target binding by generating conformation-changing sequences or even primer-free SELEX (Wang, 2020; Lai \& DeStefano, 2012). Other structural features normally amenable to SELEX process involve high GC nucleotides and low nucleotide branching (Komarova \& Kuznetsov, 2019; Vorobyeva et al., 2018). Nonetheless, sequences with predetermined patterns, like stem-loops, G-quadruplexes, or three-way junctions have replaced the need for uniform nucleotide distribution while designing libraries for special target proteins. Chemical modification of nucleotides, a fairly new advancement, adds to the multi-dimensional aspect of SELEX library compilation (Komarova \& Kuznetsov, 2019). Whether it is adapting the sugar-phosphate

\footnotetext{
${ }^{1}$ Doping is defined as the method of introducing a metal ion called dopant (lanthanide or actinide in the text example) into the already existing nanocrystal structure of another metal to enhance optical or electrochemical properties of the latter.

2 Photo-blinking is defined as intermittent emission of fluorescent signals seen during continuous excitation of a fluorophore.
} 
backbone to have greater thermal stability and nuclease resistance or altering the bases with different moieties to expand the binding potential of a starter library, nucleotide modification offers many advantages. To learn more about post-SELEX modifications, one can consult exhaustive reviews on this topic by Wang et al., Emara et al., and Zhang et al. and others. (Wang et al., 2019; Gao et al., 2016; Ni et al., 2017; Kratschmer \& Levy, 2017; Lipi et al., 2016).

Conditions of the consecutive selection and isolation phases create more limitations for conducting an efficient SELEX protocol. A lower target density during the incubation step enhances the probability of selection and isolation of high-affinity aptamers and vice versa notably with greater background binding (Komarova \& Kuznetsov, 2019; Wang et al., 2012; Spill et al., 2016). Notwithstanding the low target concentration, if the number of low-affinity oligonucleotides supersedes that of the high-affinity ones, then more of the former kind are harvested during isolation phase (Komarova \& Kuznetsov, 2019, Kalra et al., 2018). Modeling software have accurately predicted that in practice, the relationship between the lower target concentration and the number of cycles to obtain high-affinity aptamers is also not linear; low concentration initially dips the required cycle number which rises steeply afterward (Komarova \& Kuznetsov, 2019; Vant-Hull et al., 1998; Seo et al., 2010; Kalra et al., 2018; Sun, Galas, \& Waterman, 1996). Optimal target concentration for selection and isolation phases is found to be equivalent to the bulk dissociation constant or $K_{D}$ of the high-affinity oligonucleotide pool (Komarova \& Kuznetsov, 2019; Spill et al., 2016; Vant-Hull et al., 1998). Each SELEX experiment designed for a new target needs to have the optimal target concentration assessed, especially the ones where the target carrier/selection medium displays high oligonucleotide binding and that are very sensitive to changes in the target level. Perfecting the selection phase conditions with a bonus negative selection step is called for while performing the whole cell-based SELEX protocol (Kalra et al., 2018; Ohuchi, 2012). The presence of targets in their native state can result in a pool of incubating oligonucleotides to other 'target-like' structures expressed on the cell. Hence, counter or negative selection that removes such pseudo-targets becomes relevant. Advanced partitioning or separation methodologies, like capillary electrophoresis, microfluidics (bead, non-bead, or sol-gel format), flow cytometry, atomic force microscopy, and surface plasmon resonance are better equipped in

\footnotetext{
${ }^{1}$ Doping is defined as the method of introducing a metal ion called dopant (lanthanide or actinide in the text example) into the already existing nanocrystal structure of another metal to enhance optical or electrochemical properties of the latter.

${ }^{2}$ Photo-blinking is defined as intermittent emission of fluorescent signals seen during continuous excitation of a fluorophore.
} 
addressing the issues faced during conventional SELEX selection step (Stoltenburg, Reinemann, \& Strehlitz 2007; Dupont et al., 2015; Ali, Elsherbiny, \& Emara, 2019).

PCR amplification and pool conditioning promote subsequent enrichment of the selected high-affinity oligonucleotides. Low target concentration is inversely proportional to the number of PCR cycles. Therefore, the greater the number of PCR cycles, the better is the yield of high-affinity oligonucleotides (Komarova \& Kuznetsov, 2019; Wang et al., 2019). Nevertheless, the caveat is that with increasing PCR cycles, the byproducts arising from template heterogeneity also accrue (Wang et al., 2019; Tolle et al., 2014; Sun, Galas, \& Waterman, 1996; Stoltenburg, Reinemann, \& Strehlitz 2007). Such sequences, mainly short, curtail amplification of desired oligonucleotides since shorter sequences are more readily amplified by PCR. Some studies have deduced 6-10 PCR amplification cycles per SELEX cycle to be adequate for a double-stranded DNA aptamer pool; exceeding this number has rather a delimiting effect as the interfering byproducts gain prominence (Komarova \& Kuznetsov, 2019). As a result, aside from target concentration and cycle numbers, primer design and concentration, type of DNA Polymerase, PCR method, and many more factors can improve SELEX output. Pool conditioning is a critical step for ensuring the quality of PCR amplified oligonucleotides (Komarova \& Kuznetsov, 2019; Stoltenburg, Reinemann, \& Strehlitz 2007; Dupont et al., 2015). For RNA aptamers, quality assurance of transcription either tied to PCR or post-amplification should be carefully monitored. Single-stranded DNA aptamers are retrieved from a cluster of double-stranded DNA oligonucleotides via separation techniques, such as enzymatic degradation of the unwanted strand, urea PAGE paired with molecular weight-based dissociation, or use of magnetic beads exploiting streptavidin-biotin interaction. Quality control of aptamers continues beyond SELEX into the characterization phase. Aptamers produced are sequenced and tested to verify their target affinity, specificity, and stability to environmental factors during application (buffer $\mathrm{pH}$, temperature, target and salt concentration, etc.) (Komarova \& Kuznetsov, 2019; Dupont et al., 2015; Ilgu et al., 2014; Campos-Fernández et al., 2020).

\subsection{DNA vs. RNA aptamers}

Due to the nature of their nucleic acid composition, DNA aptamers inherently have greater stability than their RNA counterparts (Wang et al., 2019; Zhu, Liu, \&

\footnotetext{
${ }^{1}$ Doping is defined as the method of introducing a metal ion called dopant (lanthanide or actinide in the text example) into the already existing nanocrystal structure of another metal to enhance optical or electrochemical properties of the latter.

${ }^{2}$ Photo-blinking is defined as intermittent emission of fluorescent signals seen during continuous excitation of a fluorophore.
} 
Kai, 2015). The 2'-OH group that exists on the pentose ring of ribose sugar renders RNA more susceptible to nucleases and other forms of hydrolysis than DNA. Accordingly, a starter library for RNA aptamer generation is chemically modified to increase the stability prior to the SELEX amplification step (Lipi et al., 2016). The instability of RNA structures is also reflected in their tendency to fold into different configurations with a slight alteration in the experimental conditions, thus transforming the binding affinity to its target. For instance, 23nt long neomycin Bbinding aptamer, NEO1A demonstrates variable ratios of differential binding affinity to certain aminoglycosides with a change in buffer conditions (llgu et al., 2014). On the other hand, this structural plasticity allotted to RNA aptamers proves beneficial as more structural diversity means greater probability to attain highaffinity and specificity oligonucleotides; libraries for DNA aptamers are, for this reason, made of nucleotide segments with longer and more complex randomized motifs (Vorobyeva et al., 2018; Zhu, Liu, \& Kai, 2015; Pobanz \& Lupták, 2016). The process for DNA aptamer synthesis although is known to be less time-consuming due to absence of the transcription step required for their RNA equivalent (Zhu, Liu, \& Kai, 2015; Orava, Cicmil, \& Gariépy, 2010).

\subsection{How are aptamers different from antibodies?}

Conceivably, monoclonal antibodies have become the cornerstone for biologicals in disease detection and treatment for almost four decades since their generation by Köhler and Milstein (Köhler \& Milstein, 1975). In 2017, the combined global market for diagnostic and therapeutic monoclonal antibodies was approximately worth 95bn US dollars with an average compound annual projected growth rate of 5.53\% between 2018-2026 ("Global Monoclonal Antibodies," 2018; Grilo \& Mantalaris, 2019). Despite such robust market potential, it will be reckless for the scientific community to ignore the disadvantages posed by these antibodies (Figure 1.3). Considered revolutionizing at one time, the production of monoclonal antibodies (mAbs), is a tedious and time-consuming process when compared to that of aptamer generation (Nimjee, Rusconi, \& Sullenger, 2005; Ni et al., 2020). mAbs are manufactured using a single clonal line of hybridoma cells (Köhler \& Milstein, 1975). These cells are created via hybridization of antigen primed B-lymphocytes from the spleen of inoculated mice and immortalized mouse myeloma cells. Hybridomas against a specific antigen are then propagated either in vitro or in vivo

\footnotetext{
${ }^{1}$ Doping is defined as the method of introducing a metal ion called dopant (lanthanide or actinide in the text example) into the already existing nanocrystal structure of another metal to enhance optical or electrochemical properties of the latter.

${ }^{2}$ Photo-blinking is defined as intermittent emission of fluorescent signals seen during continuous excitation of a fluorophore.
} 
(via injection into mouse peritoneal cavity) (National Research Council (US), 1999). The entire process can easily take up to 4-6 months (Ni et al., 2020). Once the nucleic acid library is designed, aptamer synthesis via conventional SELEX takes relatively shorter time (6 weeks), with refined and automated versions of this process being completed in a span of two weeks or less (Ni et al., 2020; Yang, Li, \& Gorenstein, 2011). Production of mAbs is comparatively more expensive as well, especially the cost associated with animal care. Moreover, there is the risk associated with either getting an inadequate immune response in the animal or an excessive one that causes toxicity (Nimjee, Rusconi, \& Sullenger, 2005; Ni et al., 2020). Due to their synthesis at least partially relying on an animal system, there is an inevitable batchto-batch variation and the likelihood of the existence of animal proteins (example, rodent plasma proteins) as contaminants carried over during antibody harvest. The entirely in vitro nature of aptamer generation omits occurrence of any of the abovementioned problems. In some cases, the relatively large size of mAbs (150 kDa for most immunoglobulins; $900 \mathrm{kDa}$ for IgM) inhibits their application for less accessible tissues (blood-brain barrier) or intracellular epitopes due to low membrane permeability (Mix, Goertsches, \& Zett, 2006). On the contrary, size of most aptamers ranges from 5-30 $\mathrm{kDa}$ which gives them the privilege to enter and navigate intracellular spaces to recognize deeply situated protein targets (Jayasena, 1999; Ferreira, Cheung, Missailidis, Bisland, \& Gariépy, 2009). To add to this, some studies have demonstrated intracellular or intra-organelle formation of aptamers by transfection of target cells with recombinant vectors expressing aptamer sequences under the control of a specific promoter (Meyer, Hahn, \& Rentmeister, 2011; Lakhin, Tarantul, \& Gening, 2013). One unfavorable outcome of aptamers being quite minute is their shorter half-life which stems from getting readily filtered from the kidneys though it has been established now that tagging aptamers with polyethylene glycol (PEG) can slow down the removal (Nimjee, Rusconi, \& Sullenger, 2005; Ni et al., 2020; Lakhin, Tarantul, \& Gening, 2013). The size of the antigen is another area that limits the scope of applicability of monoclonal antibodies than aptamers. Generally, antibodies can bind and mount an immune response to antigens that span between 5-30 peptides in length. Contrastingly, low molecular weight molecules (less than $1 \mathrm{kDa}$ ), also known as haptens, are difficult to detect via antibodies; hapten-antibody interaction is not immunogenic until several copies of a hapten are conjugated to a carrier protein (such as Bovine Serum Albumin) (Gefen

\footnotetext{
${ }^{1}$ Doping is defined as the method of introducing a metal ion called dopant (lanthanide or actinide in the text example) into the already existing nanocrystal structure of another metal to enhance optical or electrochemical properties of the latter.

${ }^{2}$ Photo-blinking is defined as intermittent emission of fluorescent signals seen during continuous excitation of a fluorophore.
} 
et al., 2015). Aptamers can not only recognize small molecules like ions, hormones, metabolites, etc. with ease, they are even used for discovery of targets with unspecified structure or sequence (Nimjee, Rusconi, \& Sullenger, 2005; Zheng, Zou, \& Lou, 2012; Ku, Zhang, Luo, Yen, Chen, Han, \& Lo, 2015). The most striking aspects of aptamers are heightened epitope affinity and specificity. Whereas, mAbs still suffer from cross-reactivity for closely related protein epitopes (Flores-Moreno et al., 2014). Immunogenicity has been uncovered as a serious drawback of mAbs used as therapies - host immune response can create antibodies against the administered therapeutic $\mathrm{mAbs}$ as even the purified versions could have residues of animal proteins (Harding et al., 2010; Knezevic, Kang, \& Thorpe, 2015). Host anti$\mathrm{mAbs}$ can also interfere during antigen detection. In vitro production of aptamers bears no such problems. In fact, the use of aptamers as therapeutics grants the ability to modulate the duration of a treatment (Lakhin, Tarantul, \& Gening, 2013). This is made possible through the administration of a complementary form of the aptamerdrug as an antidote that binds to the aptamer with high efficiency and stops any further aptamer-target interaction. Stability to $\mathrm{pH}$ and ambient temperature and the flexible structure that grants ease of renaturation are other properties of aptamers that make them more attractive than mAbs as therapeutic and diagnostic agents (Nimjee, Rusconi, \& Sullenger, 2005; Ni et al., 2020). Newer developments in the library designing process have further bypassed nuclease degradation of RNA aptamers with simultaneously highlighting the versatility of these biomolecules through their flexible structures (Lakhin, Tarantul, \& Gening, 2013; Vater \& Klussmann, 2015).

\footnotetext{
${ }^{1}$ Doping is defined as the method of introducing a metal ion called dopant (lanthanide or actinide in the text example) into the already existing nanocrystal structure of another metal to enhance optical or electrochemical properties of the latter.

${ }^{2}$ Photo-blinking is defined as intermittent emission of fluorescent signals seen during continuous excitation of a fluorophore.
} 


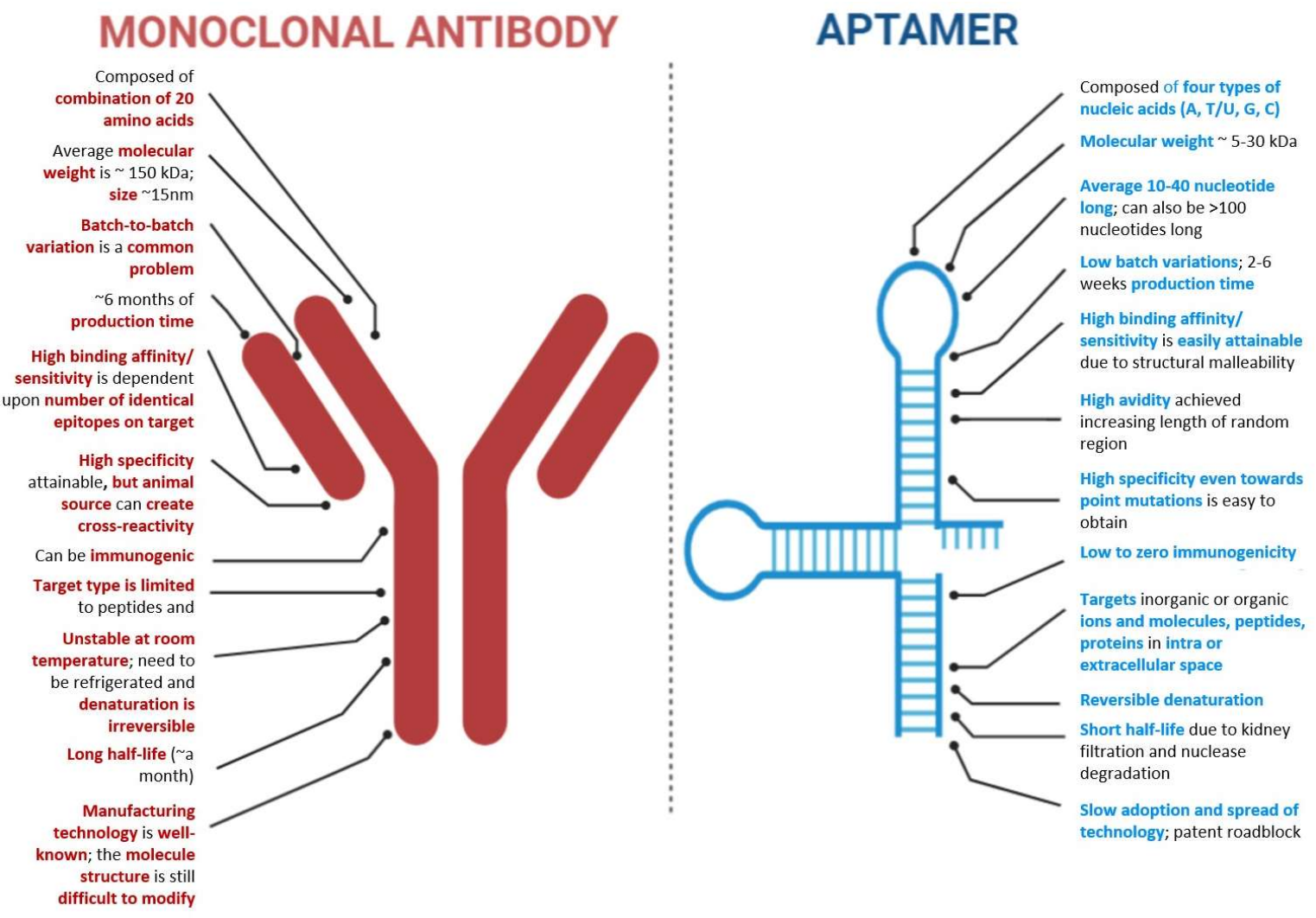

Figure 1.3 Comparison of advantages and limitations of monoclonal antibodies with those of aptamers.

\section{Application of aptamers}

With the increased popularity and utilization of biologicals as instruments for diagnosis and therapy, the shortcomings associated with monoclonal antibodies have also become more apparent. Although the core process of aptamer creation and selection was established in 1990, it took nearly two decades before studies to develop improved SELEX iterations or research to prove superiority to mAbs gained impetus (Rahimi, 2018). Inaccessibility to the SELEX technology due to patent rights, familiarity and widespread use of $\mathrm{mAb}$ production technology, burgeoning market for antibodies in disease detection and treatment were some of the reasons that diverted attention from aptamer research and development (Nimjee, Rusconi, \& Sullenger, 2005; "Global Monoclonal Antibodies," 2018; Grilo \& Mantalaris, 2019; Marks, 2012; Wade, 1980; Baird, 2010). Nevertheless, the high cost of mAb

\footnotetext{
${ }^{1}$ Doping is defined as the method of introducing a metal ion called dopant (lanthanide or actinide in the text example) into the already existing nanocrystal structure of another metal to enhance optical or electrochemical properties of the latter.

${ }^{2}$ Photo-blinking is defined as intermittent emission of fluorescent signals seen during continuous excitation of a fluorophore.
} 
production (including quality assurance), moderate antigen sensitivity as diagnostics, and low rate of success as therapeutic agents paved the way for aptamers in similar biological applications (Nimjee, Rusconi, \& Sullenger, 2005; Klutz et al., 2016; Liu, 2014). With their superior properties, aptamers are now slowly replacing $\mathrm{mAbs}$ as molecular probes for laboratory research, diagnostic agents for clinical disease detection, biosensors for environmental pollutants or contaminants, targeted drug-delivery vehicles, and therapeutic agents (Kong \& Byun, 2013; Bauer et al., 2019; Palchetti \& Mascini, 2008; Figure 1.4). Free of any trouble with data reproducibility, aptamers are being effectively used as molecular probes for cellular or sub-cellular imaging, immunolabelling (histochemistry, cell phenotyping, and protein purification), enzyme-linked sorbent assays, and lateral flow detection in research and clinical diagnostic laboratories (Bauer et al., 2019). Aptamer-based biosensors or 'aptasensors' can be conjugated to diverse reporting systems depending upon the choice of readout - from electrochemical analysis to fluorometric or colorimetric optical imaging (Song et al., 2008). These small molecule biologicals are also showing encouraging results as targeted drug-delivery agents and treatments for various ailments. One out of all the nine oligonucleotide drugs (antisense oligonucleotides, siRNAs, and aptamers) approved by FDA till 2018, is an aptamer (Macugen ${ }^{\circledR}$ for macular degeneration) with around eleven potential candidates in various phases of clinical trials (Ni et al., 2020; Kong \& Byun, 2013). For targeted drug delivery, the specific aptamers constructed are hybridized with other oligonucleotide structures, nanoparticles, or drugs. Some studies have indicated the suitability of aptamer conjugates with siRNA (AsiC) or miRNA (AmiC) for selective RNA interference (RNAi) (Ni et al., 2020; Bruno, 2013; Zhu, Niu, \& Chen, 2015; Dassie \& Giangrande, 2013). Drugs as well can be cross-linked to aptamers to limit transport to a specific cell type and minimize the bystander effect. Doxorubicin conjugates with anti-CD38 and anti-MUC1 single-stranded DNA aptamers are some examples of aptamer-based selective drug delivery (Kim et al., 2018). Chimeras formed between aptamers and distinct nanoparticle types such as gold, iron oxide, liposomes, quantum dots, dendrimers, block polymers, and more too are viewed as favorable for efficient targeted delivery (Ni et al., 2020; Bruno, 2013).

\footnotetext{
${ }^{1}$ Doping is defined as the method of introducing a metal ion called dopant (lanthanide or actinide in the text example) into the already existing nanocrystal structure of another metal to enhance optical or electrochemical properties of the latter.

2 Photo-blinking is defined as intermittent emission of fluorescent signals seen during continuous excitation of a fluorophore.
} 
Subsequent sections of this chapter elucidate how fluorescent aptamers are being conceptualized, designed, and tested for imaging, high-throughput screening, diagnosis, and treatment of pathogenic bacteria and tumor cells.

Aptamer applications

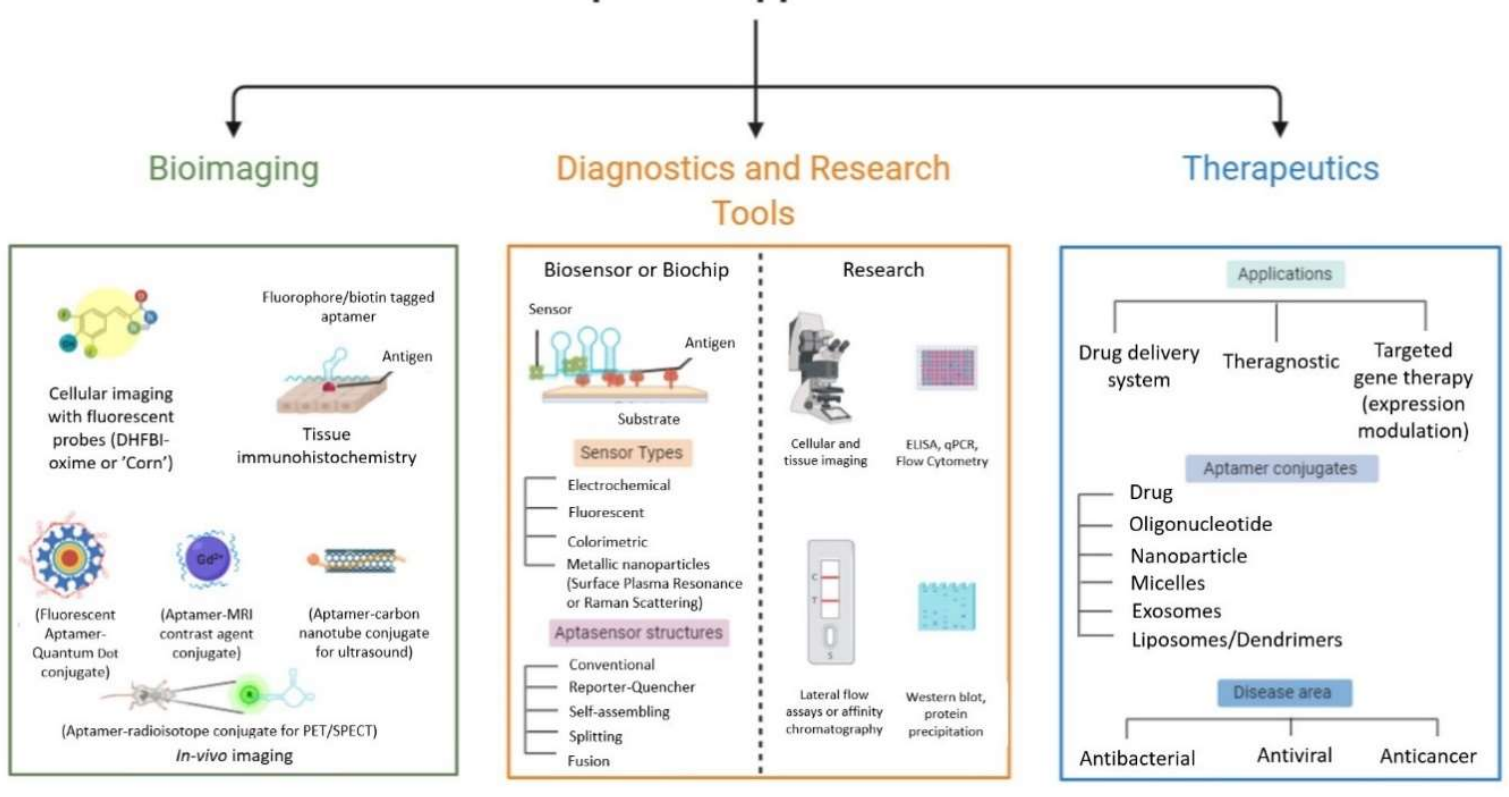

Figure 1.4 Application of aptamers. These small oligonucleotides can be used in bioimaging (some examples in the box), as diagnostic agents (biosensors or biochips), research tools, or as therapeutics (in the form of drug delivery agents, theragnostic or targeted drug delivery agents).

\section{Aptamers for pathogenic bacteria}

The global burden of infectious diseases has dramatically reduced in the $21^{\text {st }}$ century due to the implementation of effective preventive measures like rapid screening and vaccinations (Michaud, 2009; Fauci, 2001). However, resurgence of resistant strains of pathogenic microorganisms, especially bacteria and viruses, sometimes in the form of a pandemic demands enhancement of rapidity, sensitivity, specificity, and ease of use in the detection methods. Conventional diagnostic testing for pathogenic bacteria revolves around culture-based identification of microbes isolated from samples taken either from contaminated food or diseased host followed by confirmation with microscopy and biochemical phenotyping (Rajapaksha et al., 2019; Kivirand \& Rinken, 2018; Alahi \& Mukhopadhyay, 2017).

\footnotetext{
${ }^{1}$ Doping is defined as the method of introducing a metal ion called dopant (lanthanide or actinide in the text example) into the already existing nanocrystal structure of another metal to enhance optical or electrochemical properties of the latter.

${ }^{2}$ Photo-blinking is defined as intermittent emission of fluorescent signals seen during continuous excitation of a fluorophore.
} 
Desire to minimize the time lag between sample collection and reporting of test results paved the way for nucleic acid-based and immunological assays. Polymerase Chain Reaction (PCR) is the basis of assays that identify characteristic pathogen RNA or DNA. Traditional PCR and its different modalities - Real-Time PCR, multiplexed PCR, Loop-Mediated Isothermal Amplification (LAMP), Nucleic Acid Sequence-Based Amplification (NASBA), DNA microarray-provide quicker and more sensitive means of pathogen recognition (Law et al., 2015). Immunoassays hinge on the principle of antigen-antibody interaction where the antigen is a peptide, protein, or glycoprotein expressed by the pathogen. The immune-based assays have high specificity and are also used for identifying toxins released by pathogens (Law et al., 2015). Both these alternative detection techniques although require skilled personnel and sophisticated instruments, thus making them less cost-effective (Rajapaksha et al., 2019; Law et al., 2015). Moreover, due to their higher sensitivity compared to the conventional microbial cultivation techniques, these assays are more prone to generating false positives (Rajapaksha et al., 2019; Kivirand \& Rinken, 2018; Law et al., 2015).

Rapid or point-of-care diagnosis of pathogenic bacteria can be performed in a cost-effective manner via biosensor-based methods (Alahi \& Mukhopadhyay, 2017; Law et al., 2015; Byrne et al., 2009). Another advantage held by biosensor-dependent techniques over PCR and immunoassays is their ability to detect either bacterial proteins or the whole pathogen itself. A biosensor comprises two main components - bioreceptor and transducer. A bioreceptor is the pathogen recognition unit of the biosensor; this role is normally taken played by enzymes, natural or recombinant antibodies, surface receptors, nucleic acid fragments, or bacteriophages (Alahi \& Mukhopadhyay, 2017; Morales \& Halpern, 2018). A transducer converts the event of pathogen identification by the bioreceptor into a tangible signal or readout. Based on the kind of transducer, present biosensors are of optical, electrochemical, and mass-based varieties (Figure 1.5). The use of biosensors in bacteriology has expanded over the years with optical and electrochemical types being the most popular ones (Alahi \& Mukhopadhyay, 2017; Law et al., 2015; Byrne et al., 2009). Optical biosensors include Förster Resonance Energy Transfer (FRET) or flow cytometry with fluorescent probes, luminescence, surface plasma resonance (SPR) and its variations, ellipsometry, interferometry, nuclear magnetic resonance (NMR), and Raman spectroscopy (Alahi \& Mukhopadhyay, 2017; Law et al., 2015, Paniel \&

\footnotetext{
${ }^{1}$ Doping is defined as the method of introducing a metal ion called dopant (lanthanide or actinide in the text example) into the already existing nanocrystal structure of another metal to enhance optical or electrochemical properties of the latter.

${ }^{2}$ Photo-blinking is defined as intermittent emission of fluorescent signals seen during continuous excitation of a fluorophore.
} 
Noguer, 2019; Bosch et al., 2007). Optical biosensors typically offer a highly sensitive and close to real-time detection of pathogens. They also enable detection of live bacteria, but sample-preparation can be expensive and labor-intensive even for label-free SPR, NMR, or Raman spectroscopy (Alahi \& Mukhopadhyay, 2017; Law et al., 2015, Paniel \& Noguer, 2019). Electrochemical biosensors consist of amperometric, potentiometric, conductometric, and impedimetric transduction techniques that convert pathogen detection signals into alteration in current, voltage, conductance, and impedance, respectively. Being label-free, electrochemical sensors are affordable, easy to automate, and conducive for conducting testing even with large sample number; yet these are less specific than other modes of pathogen diagnosis (Alahi \& Mukhopadhyay, 2017; Law et al., 2015).

ELISA and lateral flow immunoassays (LFAs) are till date counted amongst the standard tools used for sensitive and rapid laboratory-based identification of pathogen infection, respectively (Kivirand \& Rinken, 2018; Henderson et al., 2018; Noh et al., 2019). Other than needing highly specific antibodies, these assays require sample pre-enrichment along with multiple sample preparation and data analysis steps. This overshadows their benefits of portability and ease of automation (Cho \& $\mathrm{Ku}$, 2017). Drawbacks of immunoassays continue with their recent iterations as well where antigen-antibody interactions are paired with optical or electrochemical transducers (Byrne et al., 2009). The idea behind this combination had been to create an immunological assay that has high sensitivity and specificity, fast detection and analysis, ease of use and multiplexing capabilities, and low overall operational cost. Fluorescence, luminescence, and surface plasma resonance are the top optical biosensing techniques adapted to improve conventional immunoassays (Paniel \& Noguer, 2019). Fluorescent tag-labeled antibodies (monoclonal and polyclonal) or nanoparticles are employed for flow cytometry, sandwich ELISA, and FRET whereas chemiluminescence is commonly used in other immunoassays like LFA. A fluorophore-tagged antibody can be more unstable than its unlabeled counterpart (Schuster et al., 2020). This is further complicated by low sensitivity due to sample dilution in flow cytometry or increased probability of antigen-antibody steric hindrance in sandwich ELISA (Mori \& Katayama, 2019; Sakamoto et al., 2018). FRET indicates antigen-antibody interaction by generating a shift in fluorescence intensity or lifetime with a change in distance between fluorophore donor-acceptor pair. The effectiveness of FRET to sense real-time spatiotemporal interaction in antigen-

\footnotetext{
${ }^{1}$ Doping is defined as the method of introducing a metal ion called dopant (lanthanide or actinide in the text example) into the already existing nanocrystal structure of another metal to enhance optical or electrochemical properties of the latter.

${ }^{2}$ Photo-blinking is defined as intermittent emission of fluorescent signals seen during continuous excitation of a fluorophore.
} 
antibody complex relies on a strict selection of donor/acceptor fluorophore pairs tagged onto antibodies that are placed less than $10 \mathrm{~nm}$ apart (Bajar et al., 2016). For instance, Salmonella typhimurium is discerned when a FRET donor, Alexa Fluor 546, labeled antibody (against the pathogen) and a FRET acceptor, Alexa Fluor 594, labeled protein $G$ come closer than the designated Förster radius due to conformational shift upon binding of the concerned bacteria to the donor-labeled antibody (Ko \& Grant, 2003). The tedious process of antibody labeling (including donor-acceptor pair optimization), appropriate probe selection, and signal interference from within the sample prevent FRET from being a standalone method for swift diagnosis of pathogens (Leavesley \& Rich, 2016). LFA readouts are usually colorimetric; they include a narrow strip (with plastic backing) segregated into narrow zones of different matric material (Law et al., 2015; Paniel \& Noguer, 2019). All the reagents are immobilized onto respective zones - sample application pad, conjugate area, testing zone with the test and control reagents on nitrocellulose membrane, and absorption pad. The sample moves via capillary action to the testing zone where colorimetric indicators, generally in the form of gold nanoparticles, yield the result. Photoluminescence probes, such as titanium dioxide or graphene oxide obviate the requirement of a secondary antibody and enhance the signal intensity compared to regular LFA (Paniel \& Noguer, 2019). Nevertheless, this cost-effective, simpler, user-friendly, point-of-care test can have batch-to-batch variation and drop in sensitivity at low analyte concentrations that ask for sample pre-treatment (Paniel \& Noguer, 2019; Cho \& Ku, 2017). Immunoassays with electrochemical sensors impart quantitative readout but fall short of being ideal for diagnostic analysis of pathogenic bacteria due to disadvantages presented by antibodies as the biorecognition element.

Aptamers are being intuitively developed to replace antibodies and overcome the limitations of immunological detection of pathogens. Aptamers coupled to optical transducers, also known as optical aptasensors, guarantee a wide selection of probes applicable for pathogen detection (Kivirand \& Rinken, 2018; Paniel \& Noguer, 2019). Such probes rely on either measurement of disparate properties of light - refractive index (surface plasma resonance), photon scattering by molecular vibrations (surface-enhanced Raman spectroscopy), emission of light by an electron moving from higher to lower energy state (fluorescence and chemiluminescence)or color change produced due to a chemical reaction when an analyte binds its

\footnotetext{
${ }^{1}$ Doping is defined as the method of introducing a metal ion called dopant (lanthanide or actinide in the text example) into the already existing nanocrystal structure of another metal to enhance optical or electrochemical properties of the latter.

${ }^{2}$ Photo-blinking is defined as intermittent emission of fluorescent signals seen during continuous excitation of a fluorophore.
} 
sensor (Baeyens et al., 1998; Lichtman \& Conchello, 2005; Schasfoort, 2017). Additionally, by having aptamers, as biorecognition elements, coupled to various nanoparticles in an aptasensor, the use of antibodies as signal transducers is obviated. Gold nanoparticles (AuNP) and quantum dots (QD) are the nanomaterials that are most routinely found in aptasensors (Paniel \& Noguer, 2019). Yoo et al. have developed a lab-on-chip system for the detection of three food-borne bacteria (Salmonella typhimurium, Pseudomonas aeruginosa, Lactobacillus acidophilus) with a composite of specific aptamers localized onto gold enveloped silica nanoparticles (Paniel \& Noguer, 2019; Yoo, Kim, \& Lee, 2015). This label-free method utilizes the principle of change in the refractive index with simultaneous alteration in mass during sample binding to the ligand (aptamer) for extremely sensitive (detection limit $3 \times 10^{4}$ colony forming units $/ \mathrm{ml}$ ) and specific pathogen determination of small sample (3uL). In comparison, similar antibody conjugation to nanoparticles for diagnostic tests is riddled with problems, such as instability of the complex, difficult and costly antibody modification, or poor reproducibility along with higher limit of detection (Paniel \& Noguer, 2019). Aptasensors using Surface Enhanced Raman Spectroscopy (SERS) as the transduction mechanism provide an even more sensitive high-throughput detection of pathogens. Ravindranath et al., 2011 have validated its applicability for collective detection of Salmonella typhimurium, Staphylococcus aureus, and Escherichia coli O157: H7. In this system, aptamer specific for $S$. typhimurium and antibodies targeting $S$. aureus and E. coli were localized on Raman dye-labeled gold, silver, and silver-coated gold core nanoparticles which facilitated detection limit in the range of $10^{2}$ colony forming units (CFU)/ml (Paniel \& Noguer, 2019; Ravindranath, Wang, \& Irudayaraj, 2011). Similarly, electrochemical signal detectors are paired with aptasensors. Here, target-specific aptamers are immobilized onto electrodes such that target binding induces a shift in aptamer conformation which is translated into an electrochemical variation as the quantifiable endpoint (Paniel \& Noguer, 2019). This combination has both elevated sensitivity and specificity relative to the separate use of aptamer and electrochemical sensors. A label-free diagnostic test for Salmonella typhimurium was developed by Zelada-Guillén et al. where a modified aptamer covalently bound to carboxylated single-walled carbon nanotube electrodes was incorporated as the sensor (ZeladaGuillén et al., 2009). The introduction of the target near this aptasensor disrupted the covalent interaction between aptamer and carbon nanotube, thereby creating charge

\footnotetext{
${ }^{1}$ Doping is defined as the method of introducing a metal ion called dopant (lanthanide or actinide in the text example) into the already existing nanocrystal structure of another metal to enhance optical or electrochemical properties of the latter.

${ }^{2}$ Photo-blinking is defined as intermittent emission of fluorescent signals seen during continuous excitation of a fluorophore.
} 
variation on the electrode, and hence, recorded potential; the senor detected $S$. typhimurium with the limit ranging from $0.2 \mathrm{CFU} / \mathrm{mL}$ to $106 \mathrm{CFU} / \mathrm{mL}$ in a span of 60 seconds.

\subsection{Fluorescent aptamers in the diagnosis of pathogenic bacteria: imaging and high-throughput sequencing}

Fluorescent transducers are prevalent as research and diagnostic tools. Their presence in aptasensors serves as an affordable, sensitive, faster, and reliable visual indicator of pathogen presence that can be quantified as well. To add to it, fluorescent aptasensors offer greater sensitivity and specificity range compared to colorimetric, UV, and infrared equivalents (Paniel \& Noguer, 2019; Gaudin, 2020). Yingfu Li and his group adapted a DNA aptamer (RFD-EC1) that cleaves RNA embedded in a DNA chain flanked by a fluorophore and a quencher to measure $E$. coli K12 content (Aguirre et al., 2013). It is a simple two-step process requiring the mixing of RFD-EC1 with the bacterial suspension for 35 minutes followed by readout documentation. The introduction and binding E. coli sensing DNA segment of RFD-EC1 activates the RNA cleaving activity and inhibits quencher to release fluorophore emission. This method avoids isolation of target pathogen from a mixture of other bacteria before identification, therefore, warranting ease of use. Fluorophores can be tagged onto nanoparticles conjugated to DNA/RNA aptamers for tracking and imaging bacteria. This was tested in a study where the fluoresceinlabeled trimethyl chitosan nanoparticles were first generated and then conjugated to a DNA aptamer targeting E. coli (Zhao, Lu, Yang, \& Zhang 2020). The positive charge of trimethyl chitosan nanoparticles aided the movement of negatively charged aptamer across biological membranes which was requisite for studying the movement of bacteria crossing the host barrier to fight invading infections. Singlestranded DNA aptamers tagged to fluorophores have also found utility as probes for FlSH (Fluorescent in-situ Hybridization) assay to detect pathogens like $P$. aeruginosa (Wang et al., 2011).

Quantum dots (QDs) and upconversion nanoparticles (UCNPs) have gained more popularity over fluorophores for generating fluorescent aptasensors (Li et al., 2020; Lu et al., 2017). QDs are composed of nanoscale semiconductor crystals in which electrons and holes are spaced very closely (Pisanic II, Zhang, \& Wang, 2014; Zrazhevskiy, Sena, \& Gao, 2010). Such a three-dimensional space limitation deviates the bandgap energies from those of the bulk semiconductors. QDs emit, in a size-

\footnotetext{
${ }^{1}$ Doping is defined as the method of introducing a metal ion called dopant (lanthanide or actinide in the text example) into the already existing nanocrystal structure of another metal to enhance optical or electrochemical properties of the latter.

${ }^{2}$ Photo-blinking is defined as intermittent emission of fluorescent signals seen during continuous excitation of a fluorophore.
} 
dependent manner, the energy absorbed from photons that have energies greater than the semiconductor bandgap energy. UCNPs emit strong, sharp, and visible luminescent energy accumulated from serial absorption of photons (Wang et al., 2011). Two or more incident photons at a lower energy state are taken up by these nanoparticles and released as one photon of higher energy state. UCNPs are comprised of a doped ${ }^{1}$ lanthanide or actinide metal. Sequential absorption usually occurs at a near-infrared wavelength and excites activator dopants (mostly lanthanides like terbium, holmium, ytterbium) to emit at UV/vis spectrum. QDs and UCNPs enable high-intensity and stable fluorescence signals which lack fluorophores (Li et al., 2020; Lu et al., 2017; Pisanic II, Zhang, \& Wang, 2014; Zrazhevskiy, Sena, \& Gao, 2010; Wang et al., 2011). UCNPs further have the advantage of a sharper emission spectrum with zero auto-fluorescence background that makes them suitable for multiplexing (Wang et al., 2011). Budak et al., 2016 innovatively coupled QD and UCNP to DNA aptamers against S. typhimurium and S. aureus, respectively (Kurt, Yüce, Hussain, \& Budak., 2016). These functionalized aptamers which were excited at 325nm (QD) and $980 \mathrm{~nm}$ (UCNP) were then tested to show multiplexed detection of the pathogens with minimum fluorescence spectrum crosstalk. Aptamers can function as fluorescence enhancers too. RNA aptamers known as Fluorescence Light-up Aptamers or FLAP bind small molecule, cell-permeable fluorogens and boost their emission signal by manifold (Neubacher \& Hennig, 2019). Main fluorogens used have 4-hydroxybenzlidene imidazolinone (HBI), the fluorophore moiety found in eGFP, or are derived from asymmetrical cyanine dyes and fluorophore-quencher conjugate. Low photobleaching, robust expression, and brightness are a few FLAP properties that make them suitable for tracking pathogen metabolites or mRNAs in live cells. Jaffrey and co-workers exemplified this FLAP application by creating a bifunctional FLAP using Spinach fluorogen for real-time sensing and imaging of S-adenosylmethionine (SAM) and adenosine 5'-diphosphate (ADP) in E. coli (Neubacher \& Hennig, 2019; Paige et al., 2012; Song, Wenjiao, \& Jaffrey, 2014).

Aptamers can be adopted in a variety of ways for sequencing pathogenic RNA/DNA and proteins. Combining the attributes of high specificity binding of DNA aptamers to several kinds of target molecules with PCR, low concentration analytes can be readily calculated. This detection assay termed as aptamer-based regionally protected PCR (ARP-PCR) features the complex formation of the DNA aptamer and

\footnotetext{
${ }^{1}$ Doping is defined as the method of introducing a metal ion called dopant (lanthanide or actinide in the text example) into the already existing nanocrystal structure of another metal to enhance optical or electrochemical properties of the latter.

${ }^{2}$ Photo-blinking is defined as intermittent emission of fluorescent signals seen during continuous excitation of a fluorophore.
} 
its target with subsequent DNAse I treatment before amplification such that the unbound aptamer segments are cleaved (Lin \& McNatty, 2009). As a result, only the target-bound portion of the DNA aptamer is amplified and sequenced. Target analytes with concentrations as low as $10^{-14} \mathrm{~mol} / \mathrm{L}$ have been detected with ARPPCR. Increasingly, diagnostic tests are being developed in portable formats. Reducing the number of total required components and controlling enzyme activities in PCR are critical factors for ensuring effective portability. For instance, the flexible structure of an aptamer permits its usage as a Taq polymerase inhibitor for hot-start or isothermal RT-PCR; it not only reduces the reaction activation time but also prevents unwanted polymerase activity after reaction completion that could disturb the baseline readings ("Using aptamers to control enzyme activity"). Singlecell high-throughput sequencing of bacteria is a time-consuming multi-step process. Adaptation of fluorescent aptamers targeting 16S rRNA and RNA-binding proteins (RBPs) is speculated to make the process less error-prone (Tok, 2000; van Gijtenbeek \& Kok, 2017). This concept has been validated to an extent for high-throughput sequencing of secretory metabolites of engineered bacteria. Abate et al. have harnessed the power of ultrahigh-throughput droplet screening and Spinach fluorogen functionalized aptamer in the method referred to as RNA aptamer in the droplet (RAPID) (Abatemarco et al., 2017). The concentration of non-fluorescent droplet-sorted secretory metabolites is easily documented in this technique via translation of target recognition by aptamer as a facile fluorescent signal. Notwithstanding the progress in fluorescent aptamers or aptasensors, the process to reshape and appoint them for high-throughput sequencing is still in preliminary stages.

\footnotetext{
${ }^{1}$ Doping is defined as the method of introducing a metal ion called dopant (lanthanide or actinide in the text example) into the already existing nanocrystal structure of another metal to enhance optical or electrochemical properties of the latter.

${ }^{2}$ Photo-blinking is defined as intermittent emission of fluorescent signals seen during continuous excitation of a fluorophore.
} 


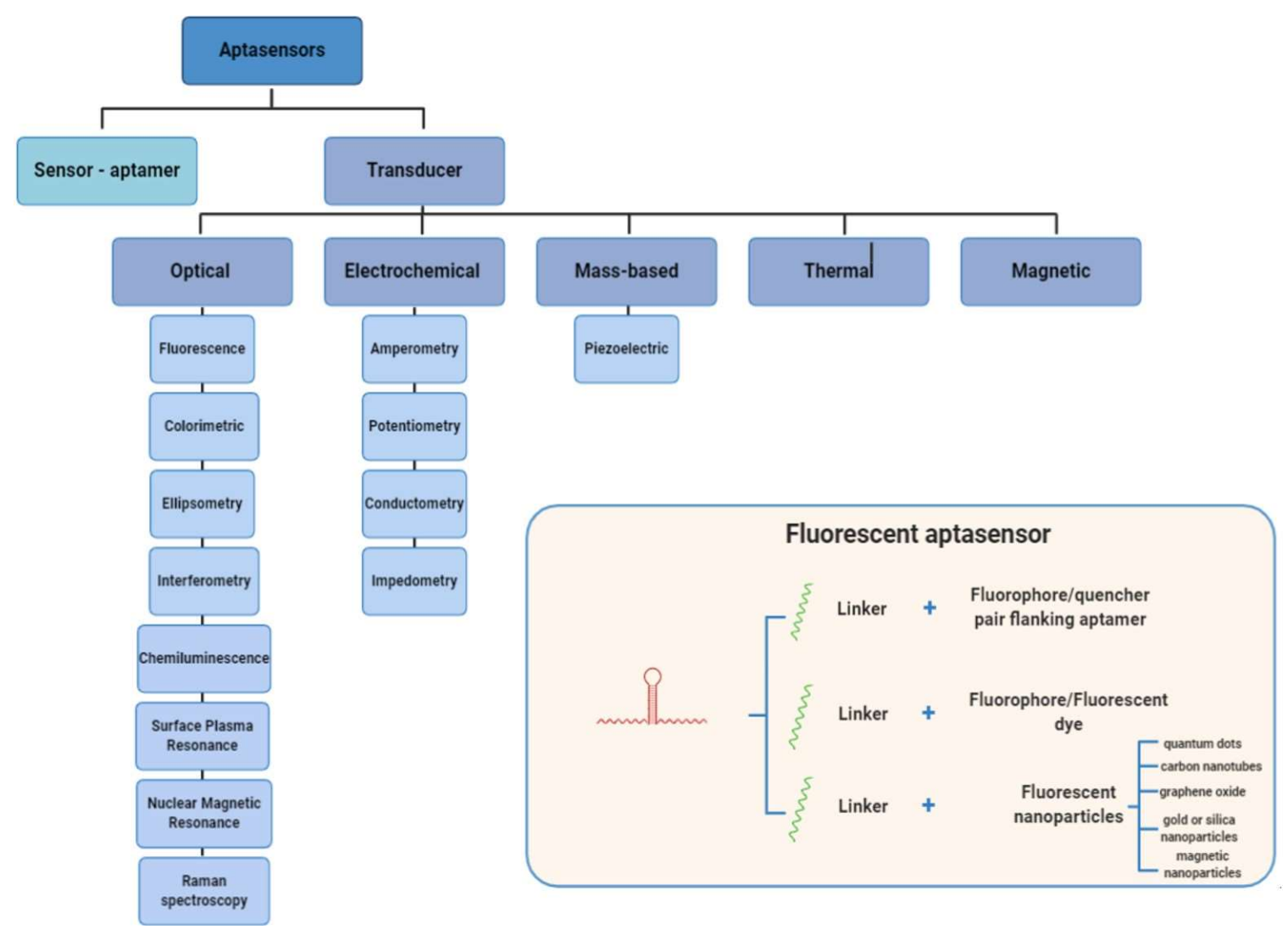

Figure 1.5 Various forms of aptasensors based on the transducer types. The box presents some ways in which a fluorescent aptamer is connected to fluorescent transducers.

\subsection{Fluorescent aptamers in the treatment against pathogenic bacteria}

Having shown their promise as diagnostic agents, aptamers are now the burgeoning as therapeutics against microbes and cancer cells. The drive to find alternatives to antibiotics is stronger than ever because of increasing resistance to these agents amongst microbes (Afrasiabi et al., 2020). Staphylococcus aureus and Pseudomonas aeruginosa are some of the prime examples of pathogenic bacteria that have developed antibiotic-resistant strains responsible for nosocomial infections (MacDougall et al., 2005). Vivekananda et al. have successfully designed and tested DNA aptamers, AR-27, AR-33, AR-36, and AR-49 for effectively recognizing alphatoxin released by $S$. aureus and inhibiting transcription of proinflammatory cytokine

\footnotetext{
${ }^{1}$ Doping is defined as the method of introducing a metal ion called dopant (lanthanide or actinide in the text example) into the already existing nanocrystal structure of another metal to enhance optical or electrochemical properties of the latter.

${ }^{2}$ Photo-blinking is defined as intermittent emission of fluorescent signals seen during continuous excitation of a fluorophore.
} 
genes, IL-17 and TNF-alpha (Tumor Necrosis Factor) to prevent cell death of Jurkat cells (Vivekananda, Salgado, \& Millenbaugh, 2014). To exemplify aptamer targeting whole bacterial cell, Chen et al. reportedly constructed a specific ssDNA aptamer against Mycobacterium tuberculosis with an association constant range of $10^{5}-10^{6} \mathrm{M}$ (Chen et al., 2007). This aptamer was shown to have antibacterial effects both in vitro and in vivo. Aptamers used as diagnostic tools against pathogens can be modulated to concomitantly function as therapeutic agents either directly as drugs or indirectly as drug targeting or delivery vehicles (Afrasiabi et al., 2020). Chandra Ray et al. built multifunctional nanoparticles for detecting and targeting multi-drug resistant (MDR) Salmonella typhimurium (Pramanik et al., 2014). These nanoparticles were made of a magnetic core with a plasmonic shell coupled to aptamer bound to methylene blue dye, and an antibody specific to MDR Salmonella typhimurium DT104 strain. The multifunctional nanoparticles were shown to be capable of selectively separating MDR Salmonella from a blood sample (via magnetic force), followed by its subsequent fluorescence imaging and killing (combined photodynamic and photothermal effect). A similar proof-of-principle theragnostic was invented by the Chandra Ray's group against methicillin-resistant S. aureus or MRSA. Resistant $P$. aeruginosa can exist in a less metabolically active state to avoid killing by antibiotics (Soundy \& Day, 2017). Therefore, to determine the microbial metabolic state with its simultaneous detection and isolation, Darren Day et al., 2017 incorporated 5-cyano2,3-ditolyl tetrazolium chloride to a mix of DNA aptamer bound bacterial cells. This compound is then converted into an insoluble fluorescent precipitate by metabolically live cells and so, one can distinguish live resistant $P$. aeruginosa cells in a biofilm with other bacterial strains. The same concept could be exploited for designing theragnostic aptamer against the microbe - a DNA aptamer of fluorophore-quencher design coupled to fluorescent nanoparticles like quantum dots could be used; the aptamer would be created against an essential pathogen enzyme such that the pathogen enzyme recognition element will be flanked by fluorophore and quencher, where binding and disruption of the enzyme function would release fluorescent signal indicating antimicrobial activity. Furthermore, the fluorescence from QD could be used without spectral interference for imaging. Although promising, the development of antibacterial aptamers as theragnostic is still in early stages; thus far, there is a dearth of preclinical studies published in this area (See Table 1.1).

\footnotetext{
${ }^{1}$ Doping is defined as the method of introducing a metal ion called dopant (lanthanide or actinide in the text example) into the already existing nanocrystal structure of another metal to enhance optical or electrochemical properties of the latter.

2 Photo-blinking is defined as intermittent emission of fluorescent signals seen during continuous excitation of a fluorophore.
} 


\section{Aptamers for tumors.}

\subsection{Fluorescent aptamers in tumor diagnosis: imaging and single-cell profiling}

A well-rounded cancer diagnosis involves quantified discernment of tumor cells and associated biomarkers. Aptamers have the structural versatility to sense whole cells and their proteins (secreted, surface-expressed or intracellular) (Nimjee, Rusconi, \& Sullenger, 2005; Jayasena, 1999; Ferreira, Cheung, Missailidis, Bisland, \& Gariépy, , 2009; Zheng, Zou, \& Lou, 2012; Ku, Zhang, Luo, Yen, Chen, Han, \& Lo, 2015). Evidently, several studies have revealed the suitability of aptasensors as new-age cancer diagnostic tools (Huang et al., 2020; Musumeci et al., 2017; Ruiz Ciancio, Vargas, Thiel, Bruno, Giangrande, \& Mestre, 2018; Yoon \& Rossi, 2018; Chang, Donovan, \& Tan, 2013). Optical aptasensors have been studied far more than electrochemical ones for distinguishing cancer cells. The most frequently designed optical sensors for cancer cell detection transduce target capture signals via fluorescence or colorimetry (Eivazzadeh-Keihan et al., 2018). There are two major ways in which currently used cancer-specific fluorescent aptamers have been generated (Musumeci et al., 2017; Eivazzadeh-Keihan et al., 2018). The first method involves coupling the aptamer to fluorescent nanoparticles, usually quantum dots. This coupling prevents the fast clearance of aptamers from the body and provides label-free fluorescence at the same time. Moreover, a mix of QDs with nonoverlapping emission spectrums conjugated to discrete aptamers against multiple cancers can be used in a multiplex detection system (Ruiz Ciancio, Vargas, Thiel, Bruno, Giangrande, \& Mestre, 2018; Kang, Chae, Cho, Lee, \& Kim,, 2009). Kimet al., 2009 presented this concept in the form of a diagnostic system that conducts parallel detection of three cancer markers - tenascin-C, nucleolin, and MUC-1 (mucin). Cheng et al., 2017 demonstrated the utility of A32 aptamer labeled with QD in preand post-operative imaging of glioma (Ruiz Ciancio, Vargas, Thiel, Bruno, Giangrande, \& Mestre, 2018; Tang, Huang, Zhang, Zhou, Tan, Pi, Pi, Cheng, Zheng, \& Cheng,., 2017). The A32 aptamer specifically binds to surface receptor EGFR (Epidermal Growth Factor Receptor) variant III that is overexpressed on glioma cells causing high-intensity fluorescence important for cancer detection. Adding QDs after aptamer-cancer cell binding precludes any negative effect of QD ligation to aptamer conformation. Wu et al., 2015 used such a labeling strategy to identify lung cancer cells (Ruiz Ciancio, Vargas, Thiel, Bruno, Giangrande, \& Mestre, 2018; Wu et

\footnotetext{
${ }^{1}$ Doping is defined as the method of introducing a metal ion called dopant (lanthanide or actinide in the text example) into the already existing nanocrystal structure of another metal to enhance optical or electrochemical properties of the latter.

2 Photo-blinking is defined as intermittent emission of fluorescent signals seen during continuous excitation of a fluorophore.
} 
al., 2015). They incubated lung carcinoma cells with specific biotin-tagged aptamer and then introduced streptavidin-coated QDs. The formation of a cancer cellaptamer-QD complex resulted in a shift in fluorescence emission wavelength. On the other hand, gold nanoparticles act as fluorescence quenchers. Their ligation to aptamers in this format has been tested in the detection of tumor marker, thrombinalpha (Liao et al., 2012; Kurseev et al., 2020). Modified TBA15, a 15-mer DNA aptamer targeting thrombin-alpha is ligated to AuNP a) by adsorption or b) by covalent interaction with its complement strand on the nanoparticle surface. Aptamerthrombin complex formation releases AuNP and relieves fluorescence inhibition. The second form of fluorescent aptasensors in cancer diagnostics are directly labeled with fluorescent tags. These include molecular beacons (fluorescent/quencher sets) or aptamers labeled with fluorescent dyes (Ruiz Ciancio, Vargas, Thiel, Bruno, Giangrande, \& Mestre, 2018). Cy5-labeled fluorescent aptamers targeting Burkitt's lymphoma cells (Ramos cells) have been used by Shi et al., 2011 for bioimaging of the tumor-bearing mice (Ruiz Ciancio, Vargas, Thiel, Bruno, Giangrande, \& Mestre, 2018; Dougherty, Cai, \& Hong, 2015; Shi et al., 2011). Such fluorescent dye linked aptamers give the ability to image tumor cells for 5-6 hours due to longer fluorescence stability. Molecular beacons are short oligonucleotide structures (aptamers in this case) that are flanked by fluorophore and quencher molecules at $5^{\prime}$ and 3', respectively. Due to the stem-loop structure, fluorophore and quencher are in close proximity to each other in the absence of the target resulting in no fluorescence. Hybridization of the target and the recognition element on the aptamer separates the fluorophore-quencher pair and activates fluorescence. $\mathrm{Zu}$ et al., 2014 modeled their aptamer identifying lymphoma circulating tumor cell (CTC) on the molecular beacon principle (Ruiz Ciancio, Vargas, Thiel, Bruno, Giangrande, \& Mestre, 2018; Zeng, Tung, \& Zu, 2014). In their study, the aptamer was developed to target specific CTC surface biomarker, ligation to which led to aptamer-reporter internalization. Once intracellular, the aptamer-reporter is transported to lysosomes and degraded, thus causing the release of the fluorophore.

There are many avenues in single-cell mRNA and genomic sequencing where the implementation of aptasensors could be beneficial. To begin with, aptamers for cancer surface antigens can be immobilized within the microfluidic device flow channels to allow efficient capturing of singular cancer cells per nano-well (Ruiz Ciancio, Vargas, Thiel, Bruno, Giangrande, \& Mestre, 2018). Philips et al., 2009 were

\footnotetext{
${ }^{1}$ Doping is defined as the method of introducing a metal ion called dopant (lanthanide or actinide in the text example) into the already existing nanocrystal structure of another metal to enhance optical or electrochemical properties of the latter.

${ }^{2}$ Photo-blinking is defined as intermittent emission of fluorescent signals seen during continuous excitation of a fluorophore.
} 
one of the first to experimentally illustrate the proof-of-concept for it (Phillips et al., 2009). They used a modified Sgc8 aptamer against the T-cell Acute Lymphocytic Leukemia cell line, CCRF-CEM; fluorescein was crosslinked at the 5' end for visualization, and biotin was tagged at the $3^{\prime}$ end to immobilize aptamer onto a streptavidin-coated plate. The system captured cancer cells with 97 percent purity and 80 percent efficiency. Aptamers have also been hypothesized to be used for biomarker profiling of a large number of cancer cells in a cell-SELEX format (Eivazzadeh-Keihan et al., 2018; Pang et al., 2018). Nonetheless, comparable to the situation with antibacterial aptamers, the ones targeting cancer cells have not been studied well so far for their capabilities in biomarker discovery and high-throughput screening (Pang et al., 2018; Chang, Donovan, \& Tan, 2013; Doud \& Woyke, 2017).

\subsection{Aptamers as anti-cancer therapeutics}

Theragnostic and targeted drug delivery agents dominate the roles that aptamers serve as anticancer therapeutics (Fu \& Xiang, 2020; Wu et al., 2015; Zhang, Hong, \& Cai, 2011). In their drug carrier form, aptamers can be coupled to small molecules or cytotoxic agents, special inhibitory RNAs (siRNA, miRNA, lncRNA), or nanoparticles (liposomes, dendrimers, etc.) loaded with anticancer drugs (Zhou et al., 2009; Liu et al., 2014). Chemotherapeutics like doxorubicin have been conjugated with cancer cell targeting aptamers in various scientific investigations. In one of their prostate cancer studies, Bagalkot et al., 2006 explored the possibility of successfully delivering doxorubicin to tumor cells by complexing the cytotoxic drug molecules with the internally hybridized double-stranded RNA aptamer A10 (Zhang, Hong, \& Cai, 2011; Bagalkot et al., 2006). This aptamer was previously validated for specific binding to prostate-specific membrane antigen (PSMA) which is found selectively overexpressed on prostate cancer cells. PSMA has an internalization signal that is activated upon protein binding to the antigen on the cell surface, ultimately resulting in endosomal uptake of the bound protein. Taking advantage of this PSMA feature, doxorubicin intercalated to GC rich region of A10 aptamer was then shown to only target PMSA positive $\mathrm{LNCaP}$ and spare the antigen negative PC3 prostate cancer cells in vitro. Aptamer covalently tagged to doxorubicin molecules via a hydrazone linker has in a similar way been tested to determine its effectiveness in targeting acute lymphoblastic leukemia T cells (T-ALL) (Zhang, Hong, \& Cai, 2011; Huang et al., 2009). Methotrexate (MTX) has also been

\footnotetext{
${ }^{1}$ Doping is defined as the method of introducing a metal ion called dopant (lanthanide or actinide in the text example) into the already existing nanocrystal structure of another metal to enhance optical or electrochemical properties of the latter.

${ }^{2}$ Photo-blinking is defined as intermittent emission of fluorescent signals seen during continuous excitation of a fluorophore.
} 
covalently conjugated to a DNA aptamer CD117, which contains a G-quadruplex structure, via N-hydroxysuccinimide (NHS) mediated amine coupling; this aptamer effectively inhibited AML cell growth (Hori et al., 2018). Cell internalizing aptamers can be efficiently conjugated to anticancer RNAs (Yoon \& Rossi, 2018). The first PSMA targeting aptamer-siRNA complex was reportedly tested in 2006 (Chu et al., 2006). Recent studies have found EpCAM (epithelial cell adhesion molecule) targeting aptamer-siRNAs becoming attractive candidates for future treatment agents targeting epithelial cancers and related circulating tumor cells (Hori et al., 2018; Subramanian et al., 2015). Using the breast cancer cell line MCF-7 based xenograft mouse model, Subramaniam et al., 2015 have evidenced the effective and selective cytotoxicity of the EpCAM-aptamer-siRNA complex. The complex was assembled to help internalization of siRNA against polo-like kinase (PLK1) via cell surface binding of EpCaM, a cancer biomarker. EpCaM ASiC can be potent drugs against circulating tumor cells too (Hori et al., 2018; Gilboa-Geffen et al., 2015; Wang et al., 2015). EpCaM ASiC was also developed against survivin, a pro-survival or anti-apoptotic molecule overexpressed in Dox-resistant breast CTCs (Hori et al., 2018; Wang et al., 2015). By administering this chimera to a breast cancer xenograft model, the group exhibited survivin knockdown and subsequent killing circulating tumor cells (CTCs) due to the reversal of Dox resistance.

By conjugating an RNA aptamer, GL21.T that targets Axl receptor tyrosine kinase, to miRs like let-7g or miR-212, Esposito et al., 2014 and Iaboni et al., 2016 have separately provided strong evidence for their growth inhibitory and TRAIL (TNFalpha Related Apoptosis Inducing Ligand) mediated cell death in lung cancer cells, respectively (Hori et al., 2018; Iaboni et al., 2016; Esposito et al., 2014). Liposomes are the most prevalent form of nanoparticles complexed with aptamers for targeting cancer cells (Liu et al., 2014). An FDA approved PEGylated liposomal form of doxorubicin called Doxil has been adapted by Xing et al., 2013 in an anticancer aptamer form to increase its specific toxicity against breast cancer cells (Liu et al., 2014; Xing et al., 2013). Doxil, as is provides a decent selectivity in cancer cells. However, a lack of a cancer target attached to this drug-loaded liposome may be risky for use in patients due to unwanted toxicity in normal cells. Therefore, Doxil was conjugated to AS1411, aptamer targeting nucleolin (overexpressed on cancer cells) through covalent binding with a spacer molecule. The study convincingly showed enhanced efficacy and selectivity of Dox cytotoxicity in the aptamer form

\footnotetext{
${ }^{1}$ Doping is defined as the method of introducing a metal ion called dopant (lanthanide or actinide in the text example) into the already existing nanocrystal structure of another metal to enhance optical or electrochemical properties of the latter.

${ }^{2}$ Photo-blinking is defined as intermittent emission of fluorescent signals seen during continuous excitation of a fluorophore.
} 
relative to unconjugated liposomes. Aptamers by themselves can be produced as immune modulators which have a vast potential in the field of cancer therapy (Pastor et al., 2018). For instance, RNA aptamers that target and modulate the function of immune checkpoint proteins like CTLA-4 (cytotoxic T-Lymphocyte associated protein-4) or PD-1 could prevent the spread of invasive cancer cells and complement the effects of antitumor agents by improving anticancer T-cell immunity.

Theragnostic anticancer agents potentially have a great future due to their feasibility related to multifunctional capabilities. Akin to antibacterial aptasensors used as theragnostic, the anticancer aptasensors too commonly have fluorescent transducers (Wu et al., 2015). Daniel Elson's group combined gold nanorods with a Cy5 (a near-infrared fluorophore) tagged AS1411 aptamer to create an aptasensor for targeted imaging and treatment of cancer cells (Gallina et al., 2016). Once inside a cell, gold nanorods can be activated using near-infrared light causing them to emit heat in the local environment to the extent of killing their host cell. To maximize such a special property of phototherapy for cancer cell death, the group crosslinked an internalizing aptamer targeting AS1411. The resulting product essentially exhibited superior fluorescence for imaging cancer cells (HeLa) and provided a new mode of selectively killing chemotherapy-resistant tumor cells. Quantum dots have better photostability and less photobleaching compared to fluorescent dyes and have wider absorption but narrow emission spectra (Pisanic II, Zhang, \& Wang, 2014). A complex of a chemotherapeutic drug, an RNA aptamer, and a QD, Dox-A10 has been positively tested by Farokhzad et al., 2007 for use as a cancer imaging tool and therapy in prostate cancer cells (Bagalkot, Zhang, Levy-Nissenbaum, Jon, Kantoff, Langer, \& Farokhzad, 2007). The key characteristic of this theragnostic is based on the principle of Bi-FRET- a donor-acceptor interaction between QD and Dox and a donor-quencher relation between Dox and the aptamer (here targeting PSMA). Internalization of this complex into intracellular space (endolysosomes) and the release of doxorubicin from the complex releases the fluorescence inhibition on both the drug and QD. This assists in clearly visualizing both drug delivery and the release inside the target cell. UPNCs or upconverting nanoparticles have greater photostability, fluorescent signal intensity, biocompatibility, and chemical stability. Moreover, they do not have a photo-blinking ${ }^{2}$ problem and can be cleared from the body in 7 days. (Wang, Abbineni, Clevenger, Mao, \& Xu, 2011) Hence, they make an

\footnotetext{
${ }^{1}$ Doping is defined as the method of introducing a metal ion called dopant (lanthanide or actinide in the text example) into the already existing nanocrystal structure of another metal to enhance optical or electrochemical properties of the latter.

${ }^{2}$ Photo-blinking is defined as intermittent emission of fluorescent signals seen during continuous excitation of a fluorophore.
} 
excellent choice for developing anticancer theragnostic agents. The feature of tuneability in a few aptamers being presently investigated grants them a significant therapeutic edge over their predecessors (Gray et al., 2018). Sullenger et al., 2005 identified an RNA aptamer, E3 that is selectively internalized by prostate cancer cells and first complexed it with maleimide-DyLight 650 (DL650; fluorescent dye) (Nimjee, Rusconi, \& Sullenger, 2005). Monomethyl auristatin E (MMAE) and monomethyl auristatin F (MMAF), potent cytotoxic agents, were then separately ligated to the fluorescent E3 aptamer. The resultant complex allowed effective visualization of targeted drug delivery both in vivo and in vitro. To add to its appeal even further, they also designed antisense oligonucleotides to these aptamer conjugates for neutralization of the cytotoxic activity in case of unwanted effects on normal tissue. There are a plethora of improved diagnostic and treatment options that fluorescent theragnostic aptamers have brought to the existing arsenal of anticancer agents (See Table 1.1). Despite that, the number of such agents passing clinical trials is paltry compared to their antibody counterparts (Kaur, Bruno, Kumar, \& Sharma, 2018).

\section{Conclusion}

Aptamers are multipurpose small oligonucleotides that were first ideated and produced four decades ago (Sullenger, Gallardo, Ungers, \& Gilboa, 1990; Tuerk \& Gold, 1990). Efforts to revive aptamer research and utilize it for diagnostic and therapeutic purposes has gained momentum in recent years (Gaudin, 2020). Progress has been made to improve and customize the SELEX process per the properties required in an aptamer (Lipi et al., 2016). Although monoclonal antibodies and their improved chimeras (e.g. bispecific and humanized antibodies) are in demand till date, their relatively longer and costlier production, stability, and immunotoxicity during application along with other problems mentioned earlier make them less suitable for use in diagnostic kits as well as therapies (Lu et al., 2020; Helwick C, 2020; Glassy \& Gupta, 2014; Chames et al., 2009). Contrastingly, the relative ease of production, the feasibility of synthesis to several conditions (including in vivo), the ability to target a varied range of biomolecules, and pliability in the structure are some of the many reasons that favor investing in aptamer development (Tantirungrotechai \& Soontornworajit, 2016; Chinnappan et al., 2020; Zheng, Zou, \& Lou, 2012). A continuous decrease in the cost of high-throughput

\footnotetext{
${ }^{1}$ Doping is defined as the method of introducing a metal ion called dopant (lanthanide or actinide in the text example) into the already existing nanocrystal structure of another metal to enhance optical or electrochemical properties of the latter.

${ }^{2}$ Photo-blinking is defined as intermittent emission of fluorescent signals seen during continuous excitation of a fluorophore.
} 
32 of 52

sequencing and its wider availability also have boosted aptamer research (Pereira \& Williams, 2007). In particular, fluorescent aptamers are being studied more since they have a large potential for being utilized as theragnostic agents. Arguably, pairing with fluorescent probes or nanoparticles may even help in mitigating the issue of shorter tissue half-life faced by aptamers (Catuogno, Esposito, \& de Franciscis, 2016).

Conflicts of Interest: The authors declare no conflicts of interest.

\section{References}

Abatemarco, J., Sarhan, M. F., Wagner, J. M., Lin, J.-L., Liu, L., Hassouneh, W., Yuan, S.-F., Alper, H. S., \& Abate, A. R. (2017). RNA-aptamers-in-droplets (Rapid) high-throughput screening for secretory phenotypes. Nature Communications, 8(1), 1-9.

Afrasiabi, S., Pourhajibagher, M., Raoofian, R., Tabarzad, M., \& Bahador, A. (2020). Therapeutic applications of nucleic acid aptamers in microbial infections. Journal of Biomedical Science, 27.

Aguirre, S., Ali, M., Salena, B., \& Li, Y. (2013). A sensitive dna enzyme-based fluorescent assay for bacterial detection. Biomolecules, 3(4), 563-577.

Ali MH, Elsherbiny ME, Emara M. Updates on Aptamer Research. Int. J. Mol. Sci. 2019, 20(10), 2511.

Aquino-Jarquin, G., \& Toscano-Garibay, J. D. (2011). Rna aptamer evolution: Two decades of selection. International Journal of Molecular Sciences, 12(12), 9155-9171.

Arora, A., Sunbul, M., \& Jäschke, A. (2015). Dual-colour imaging of RNAs using quencher- and fluorophorebinding aptamers. Nucleic Acids Research, 43(21), e144.

Baeyens, W. R. G., Schulman, S. G., Calokerinos, A. C., Zhao, Y., García Campaña, A. M., Nakashima, K., \& De Keukeleire, D. (1998). Chemiluminescence-based detection: Principles and analytical applications in flowing streams and in immunoassays. Journal of Pharmaceutical and Biomedical Analysis, 17(6-7), 941953.

Bagalkot, V., Farokhzad, O. C., Langer, R., \& Jon, S. (2006). An aptamer-doxorubicin physical conjugate as a novel targeted drug-delivery platform. Angewandte Chemie International Edition, 45(48), 8149-8152.

Bagalkot, V., Zhang, L., Levy-Nissenbaum, E., Jon, S., Kantoff, P. W., Langer, R., \& Farokhzad, O. C. (2007). Quantum dot-aptamer conjugates for synchronous cancer imaging, therapy, and sensing of drug delivery based on bi-fluorescence resonance energy transfer. Nano Letters, 7(10), 3065-3070.

Baird, G. S. (2010). Where are all the aptamers? American Journal of Clinical Pathology, 134(4), 529-531.

${ }^{1}$ Doping is defined as the method of introducing a metal ion called dopant (lanthanide or actinide in the text example) into the already existing nanocrystal structure of another metal to enhance optical or electrochemical properties of the latter.

${ }^{2}$ Photo-blinking is defined as intermittent emission of fluorescent signals seen during continuous excitation of a fluorophore. 
Bajar, B., Wang, E., Zhang, S., Lin, M., \& Chu, J. (2016). A guide to fluorescent protein fret pairs. Sensors, 16(9), 1488.

Bauer, M., Strom, M., Hammond, D. S., \& Shigdar, S. (2019). Anything you can do, i can do better: Can aptamers replace antibodies in clinical diagnostic applications? Molecules, 24(23), 4377.

Bing, T., Zheng, W., Zhang, X., Shen, L., Liu, X., Wang, F., Cui, J., Cao, Z., \& Shangguan, D. (2017). Triplexquadruplex structural scaffold: A new binding structure of aptamer. Scientific Reports, 7(1), 15467.

Blind, M., \& Blank, M. (2015). Aptamer selection technology and recent advances. Molecular Therapy Nucleic Acids, 4, e223.

Bosch, M., Sánchez, A., Rojas, F., \& Ojeda, C. (2007). Recent development in optical fiber biosensors. Sensors, 7(6), 797-859.

Bruno, J. (2013). A review of therapeutic aptamer conjugates with emphasis on new approaches. Pharmaceuticals, 6(3), 340-357.

Burgert, H.-G., Ruzsics, Z., Obermeier, S., Hilgendorf, A., Windheim, M., \& Elsing, A. (2002). Subversion of host defense mechanisms by adenoviruses. Current Topics in Microbiology and Immunology, 269, 273-318.

Byrne, B., Stack, E., Gilmartin, N., \& O’Kennedy, R. (2009). Antibody-based sensors: Principles, problems and potential for detection of pathogens and associated toxins. Sensors, 9(6), 4407-4445.

Campos-Fernández, E., Barcelos, L. S., Souza, A. G., Goulart, L. R., \& Alonso-Goulart, V. (2020). Post-selex optimization and characterization of a prostate cancer cell-specific aptamer for diagnosis. ACS Omega, 5(7), 3533-3541.

Carvalho, J., Paiva, A., Cabral Campello, M. P., Paulo, A., Mergny, J.-L., Salgado, G. F., Queiroz, J. A., \& Cruz, C. (2019). Aptamer-based targeted delivery of a g-quadruplex ligand in cervical cancer cells. Scientific Reports, 9(1), 1-12.

Catuogno, S., Esposito, C. L., \& de Franciscis, V. (2016). Aptamer-mediated targeted delivery of therapeutics: An update. Pharmaceuticals, 9(4).

Chames, P., Van Regenmortel, M., Weiss, E., \& Baty, D. (2009). Therapeutic antibodies: Successes, limitations and hopes for the future. British Journal of Pharmacology, 157(2), 220-233.

Chang, Y. M., Donovan, M. J., \& Tan, W. (2013). Using aptamers for cancer biomarker discovery. Journal of Nucleic Acids, 2013, 817350.

Chang, Y. M., Donovan, M. J., \& Tan, W. (2013, January 15). Using aptamers for cancer biomarker discovery [Review Article]. Journal of Nucleic Acids.

${ }^{1}$ Doping is defined as the method of introducing a metal ion called dopant (lanthanide or actinide in the text example) into the already existing nanocrystal structure of another metal to enhance optical or electrochemical properties of the latter.

${ }^{2}$ Photo-blinking is defined as intermittent emission of fluorescent signals seen during continuous excitation of a fluorophore. 
Chen, F., Zhou, J., Luo, F., Mohammed, A.-B., \& Zhang, X.-L. (2007). Aptamer from whole-bacterium SELEX as new therapeutic reagent against virulent Mycobacterium tuberculosis. Biochemical and Biophysical Research Communications, 357(3), 743-748.

Chi, C.-W., Lao, Y.-H., Li, Y.-S., \& Chen, L.-C. (2011). A quantum dot-aptamer beacon using a DNA intercalating dye as the FRET reporter: Application to label-free thrombin detection. Biosensors and Bioelectronics, 26(7), 3346-3352.

Chinnappan, R., AlZabn, R., Fataftah, A. K., Alhoshani, A., \& Zourob, M. (2020). Probing high-affinity aptamer binding region and development of aptasensor platform for the detection of cylindrospermopsin. Analytical and Bioanalytical Chemistry, 412(19), 4691-4701.

Cho, I.-H., \& Ku, S. (2017). Current technical approaches for the early detection of foodborne pathogens: Challenges and opportunities. International Journal of Molecular Sciences, 18(10), 2078.

Chu, T. C., Twu, K. Y., Ellington, A. D., \& Levy, M. (2006). Aptamer mediated siRNA delivery. Nucleic Acids Research, 34(10), e73.

Darmostuk, M., Rimpelova, S., Gbelcova, H., \& Ruml, T. (2015). Current approaches in SELEX: An update to aptamer selection technology. Biotechnology Advances, 33(6), 1141-1161.

Dassie, J. P., \& Giangrande, P. H. (2013). Current progress on aptamer-targeted oligonucleotide therapeutics. Therapeutic Delivery, 4(12), 1527-1546.

Dassie, J. P., Liu, X., Thomas, G. S., Whitaker, R. M., Thiel, K. W., Stockdale, K. R., Meyerholz, D. K., McCaffrey, A. P., McNamara, J. O., \& Giangrande, P. H. (2009). Systemic administration of optimized aptamer-siRNA chimeras promotes regression of PSMA-expressing tumors. Nature Biotechnology, 27(9), 839-846.

Delley, C. L., Liu, L., Sarhan, M. F., \& Abate, A. R. (2018). Combined aptamer and transcriptome sequencing of single cells. Scientific Reports, $8(1), 1-8$.

de-los-Santos-Álvarez, N., Lobo-Castañón, M. J., Miranda-Ordieres, A. J., \& Tuñón-Blanco, P. (2008). Aptamers as recognition elements for label-free analytical devices. TrAC Trends in Analytical Chemistry, 27(5), 437-446.

Doud, D. F. R., \& Woyke, T. (2017). Novel approaches in function-driven single-cell genomics. FEMS Microbiology Reviews, 41(4), 538-548.

Dougherty, C. A., Cai, W., \& Hong, H. (2015). Applications of aptamers in targeted imaging: State of the art. Current Topics in Medicinal Chemistry, 15(12), 1138-1152.

\footnotetext{
${ }^{1}$ Doping is defined as the method of introducing a metal ion called dopant (lanthanide or actinide in the text example) into the already existing nanocrystal structure of another metal to enhance optical or electrochemical properties of the latter.

${ }^{2}$ Photo-blinking is defined as intermittent emission of fluorescent signals seen during continuous excitation of a fluorophore.
} 
Duca, M., Vekhoff, P., Oussedik, K., Halby, L., \& Arimondo, P. B. (2008). The triple helix: 50 years later, the outcome. Nucleic Acids Research, 36(16), 5123-5138.

Dupont DM, Larsen N, Jensen JK, Andreasen PA, Kjems J. Characterization of aptamer-target interactions by branched selection and high-throughput sequencing of SELEX pools. Nucleic Acids Res. 2015 Dec 2; 43(21): e139.

Eivazzadeh-Keihan, R., Pashazadeh-Panahi, P., Baradaran, B., Maleki, A., Hejazi, M., Mokhtarzadeh, A., \& de la Guardia, M. (2018). Recent advances on nanomaterial based electrochemical and optical aptasensors for detection of cancer biomarkers. TrAC Trends in Analytical Chemistry, 100, 103-115.

Ellington, A. D., \& Szostak, J. W. (1990). In vitro selection of RNA molecules that bind specific ligands. Nature, 346(6287), 818-822.

Esposito, C. L., Cerchia, L., Catuogno, S., De Vita, G., Dassie, J. P., Santamaria, G., Swiderski, P., Condorelli, G., Giangrande, P. H., \& de Franciscis, V. (2014). Multifunctional aptamer-mirna conjugates for targeted cancer therapy. Molecular Therapy, 22(6), 1151-1163.

Fauci, A. S. (2001). Infectious diseases: Considerations for the 21st century. Clinical Infectious Diseases, 32(5), 675-685.

Feigon, J., Dieckmann, T., \& Smith, F. W. (1996). Aptamer structures from A to $\zeta$. Chemistry \& Biology, 3(8), 611-617.

Ferreira, C. S. M., Cheung, M. C., Missailidis, S., Bisland, S., \& Gariépy, J. (2009). Phototoxic aptamers selectively enter and kill epithelial cancer cells. Nucleic Acids Research, 37(3), 866-876.

Flores-Moreno, K., Celis-Meneses, J. S., Meneses-Ruiz, D. M., Castillo-Rodal, A. I., Orduña, P., Montiel, B. A., \& López-Vidal, Y. (2014). Potential cross-reactivity of monoclonal antibodies against clinically relevant mycobacteria: Monoclonal antibodies against mycobacteria. Clinical \& Experimental Immunology, 177(2), 454-463.

Fu, Z., \& Xiang, J. (2020). Aptamers, the nucleic acid antibodies, in cancer therapy. International Journal of Molecular Sciences, 21(8), 2793.

Futami, K., Kimoto, M., Lim, Y. W. S., \& Hirao, I. (2018). Genetic alphabet expansion provides versatile specificities and activities of unnatural-base dna aptamers targeting cancer cells. Molecular Therapy. Nucleic Acids, 14, 158-170.

Gallina, M. E., Zhou, Y., Johnson, C. J., Harris-Birtill, D., Singh, M., Zhao, H., Ma, D., Cass, T., \& Elson, D. S. (2016). Aptamer-conjugated, fluorescent gold nanorods as potential cancer theradiagnostic agents. Materials Science and Engineering: C, 59, 324-332.

${ }^{1}$ Doping is defined as the method of introducing a metal ion called dopant (lanthanide or actinide in the text example) into the already existing nanocrystal structure of another metal to enhance optical or electrochemical properties of the latter.

${ }^{2}$ Photo-blinking is defined as intermittent emission of fluorescent signals seen during continuous excitation of a fluorophore. 
Gao, S., Zheng, X., Jiao, B., \& Wang, L. (2016). Post-SELEX optimization of aptamers. Analytical and Bioanalytical Chemistry, 408(17), 4567-4573.

Gaudin, V. (2020). The growing interest in development of innovative optical aptasensors for the detection of antimicrobial residues in food products. Biosensors, 10(3).

Gefen, T., Vaya, J., Khatib, S., Rapoport, I., Lupo, M., Barnea, E., Admon, A., Heller, E. D., Aizenshtein, E., \& Pitcovski, J. (2015). The effect of haptens on protein-carrier immunogenicity. Immunology, 144(1), 116126.

Gilboa-Geffen, A., Hamar, P., Le, M. T. N., Wheeler, L. A., Trifonova, R., Petrocca, F., Wittrup, A., \& Lieberman, J. (2015). Gene knockdown by epcam aptamer-sirna chimeras suppresses epithelial breast cancers and their tumor-initiating cells. Molecular Cancer Therapeutics, 14(10), 2279-2291.

Glassy, M. C., \& Gupta, R. (2014). Technical and ethical limitations in making human monoclonal antibodies (An overview). Methods in Molecular Biology (Clifton, N.J.), 1060, 9-36.

Global Monoclonal Antibodies Market - by Type, Application, Region - Market Size, Demand Forecasts, Company Profiles, Industry Trends and Updates (2017-2023). 2018. Business Wire.

Gold, L. (2015). Selex: How it happened and where it will go. Journal of Molecular Evolution, 81(5-6), 140143.

Gray, B. P., Kelly, L., Ahrens, D. P., Barry, A. P., Kratschmer, C., Levy, M., \& Sullenger, B. A. (2018). Tunable cytotoxic aptamer-drug conjugates for the treatment of prostate cancer. Proceedings of the National Academy of Sciences, 115(18), 4761-4766.

Grilo, A. L., \& Mantalaris, A. (2019). The increasingly human and profitable monoclonal antibody market. Trends in Biotechnology, 37(1), 9-16.

Harding, F. A., Stickler, M. M., Razo, J., \& DuBridge, R. (2010). The immunogenicity of humanized and fully human antibodies: Residual immunogenicity resides in the CDR regions. MAbs, 2(3), 256-265.

Helwick C. 2020. Bispecific antibodies successes and challenges-The ASCO Post.

Henderson, W. A., Xiang, L., Fourie, N. H., Abey, S. K., Ferguson, E. G., Diallo, A. F., Kenea, N. D., \& Kim, C. H. (2018). Simple lateral flow assays for microbial detection in stool. Analytical Methods, 10(45), 53585363.

Hidding J. (2016). A therapeutic battle: Antibodies vs. Aptamers. Nanoscience master program, 1-20

Hori, S., Herrera, A., Rossi, J. J., \& Zhou, J. (2018). Current advances in aptamers for cancer diagnosis and therapy. Cancers, 10(1).

${ }^{1}$ Doping is defined as the method of introducing a metal ion called dopant (lanthanide or actinide in the text example) into the already existing nanocrystal structure of another metal to enhance optical or electrochemical properties of the latter.

${ }^{2}$ Photo-blinking is defined as intermittent emission of fluorescent signals seen during continuous excitation of a fluorophore. 
Huang, R., He, L., Li, S., Liu, H., Jin, L., Chen, Z., Zhao, Y., Li, Z., Deng, Y., \& He, N. (2020). A simple fluorescence aptasensor for gastric cancer exosome detection based on branched rolling circle amplification. Nanoscale, 12(4), 2445-2451.

Huang, Y.-F., Shangguan, D., Liu, H., Phillips, J. A., Zhang, X., Chen, Y., \& Tan, W. (2009). Molecular assembly of an aptamer-drug conjugate for targeted drug delivery to tumor cells. Chembiochem: A European Journal of Chemical Biology, 10(5), 862-868.

Iaboni, M., Russo, V., Fontanella, R., Roscigno, G., Fiore, D., Donnarumma, E., Esposito, C. L., Quintavalle, C., Giangrande, P. H., de Franciscis, V., \& Condorelli, G. (2016). Aptamer-mirna-212 conjugate sensitizes nsclc cells to trail. Molecular Therapy. Nucleic Acids, 5(3), e289.

Ilgu M, Fulton BD, Yennamalli RM, Lamm MH, Sen TZ, Nilsen-Hamilton M. An adaptable pentaloop defines a robust neomycin-B RNA aptamer with conditional ligand-bound structures.RNA. 2014 Jun; 20(6): 815-824.

Jayasena, S. D. (1999). Aptamers: An emerging class of molecules that rival antibodies in diagnostics. Clinical Chemistry, 45(9), 1628-1650.

Jayasena, S. D. (1999). Aptamers: An emerging class of molecules that rival antibodies in diagnostics. Clinical Chemistry, 45(9), 1628-1650.

Kairi Kivirand \& Toonika Rinken. (2018). Biosensing technologies for the detection of pathogens-A prospective way for rapid analysis.

Kalra, P., Dhiman, A., Cho, W. C., Bruno, J. G., \& Sharma, T. K. (2018). Simple methods and rational design for enhancing aptamer sensitivity and specificity. Frontiers in Molecular Biosciences, 5, 41.

Kang, W. J., Chae, J. R., Cho, Y. L., Lee, J. D., \& Kim, S. (2009). Multiplex imaging of single tumor cells using quantum-dot-conjugated aptamers. Small, 5(22), 2519-2522.

Kanwar, J. R., Roy, K., \& Kanwar, R. K. (2011). Chimeric aptamers in cancer cell-targeted drug delivery. Critical Reviews in Biochemistry and Molecular Biology, 46(6), 459-477.

Karimi, F., \& Dabbagh, S. (2019). Gel green fluorescence ssDNA aptasensor based on carbon nanotubes for detection of anthrax protective antigen. International Journal of Biological Macromolecules, 140, 842-850.

Kaur, H., Bruno, J. G., Kumar, A., \& Sharma, T. K. (2018). Aptamers in the therapeutics and diagnostics pipelines. Theranostics, 8(15), 4016-4032.

${ }^{1}$ Doping is defined as the method of introducing a metal ion called dopant (lanthanide or actinide in the text example) into the already existing nanocrystal structure of another metal to enhance optical or electrochemical properties of the latter.

${ }^{2}$ Photo-blinking is defined as intermittent emission of fluorescent signals seen during continuous excitation of a fluorophore. 
38 of 52

Khalil, N. M., Nascimento, T. C. F. do, Casa, D. M., Dalmolin, L. F., Mattos, A. C. de, Hoss, I., Romano, M. A., \& Mainardes, R. M. (2013). Pharmacokinetics of curcumin-loaded PLGA and PLGA-PEG blend nanoparticles after oral administration in rats. Colloids and Surfaces B: Biointerfaces, 101, 353-360.

Kim, J. K., Choi, K.-J., Lee, M., Jo, M., \& Kim, S. (2012). Molecular imaging of a cancer-targeting theragnostics probe using a nucleolin aptamer- and microRNA-221 molecular beacon-conjugated nanoparticle. Biomaterials, 33(1), 207-217.

Kim, M. W., Jeong, H. Y., Kang, S. J., Jeong, I. H., Choi, M. J., You, Y. M., Im, C. S., Song, I. H., Lee, T. S., Lee, J. S., Lee, A., \& Park, Y. S. (2019). Anti-egf receptor aptamer-guided co-delivery of anti-cancer sirnas and quantum dots for theranostics of triple-negative breast cancer. Theranostics, 9(3), 837-852.

Kim, M., Kim, D.-M., Kim, K.-S., Jung, W., \& Kim, D.-E. (2018). Applications of cancer cell-specific aptamers in targeted delivery of anticancer therapeutic agents. Molecules, 23(4), 830.

Klutz, S., Holtmann, L., Lobedann, M., \& Schembecker, G. (2016). Cost evaluation of antibody production processes in different operation modes. Chemical Engineering Science, 141, 63-74.

Knezevic, I., Kang, H.-N., \& Thorpe, R. (2015). Immunogenicity assessment of monoclonal antibody products: A simulated case study correlating antibody induction with clinical outcomes. Biologicals, 43(5), 307-317.

Ko, S., \& Grant, S. A. (2003). Development of a novel FRET method for detection of Listeria or Salmonella. Sensors and Actuators B: Chemical, 96(1-2), 372-378.

Köhler, G., \& Milstein, C. (1975). Continuous cultures of fused cells secreting antibody of predefined specificity. Nature, 256(5517), 495-497.

Komarova, N., \& Kuznetsov, A. (2019). Inside the black box: What makes selex better? Molecules, 24(19), 3598.

Kong, H. Y., \& Byun, J. (2013). Nucleic acid aptamers: New methods for selection, stabilization, and application in biomedical science. Biomolecules and Therapeutics, 21(6), 423-434.

Kratschmer C, Levy M. Effect of Chemical Modifications on Aptamer Stability in Serum. Nucleic Acid Ther. 2017 Dec $1 ; 27(6)$ : 335-344.

Ku, T.-H., Zhang, T., Luo, H., Yen, T., Chen, P.-W., Han, Y., \& Lo, Y.-H. (2015). Nucleic acid aptamers: An emerging tool for biotechnology and biomedical sensing. Sensors, 15(7), 16281-16313.

Kurseev, S. A., Solovjev, A. M., Neumann, M. M., Medvedko, A. V., \& Sakharov, I. Y. (2020). Chemiluminescent and colorimetric aptamer-based assays of human $\alpha$-thrombin. Analytical Letters, 53(1), 140-151.

${ }^{1}$ Doping is defined as the method of introducing a metal ion called dopant (lanthanide or actinide in the text example) into the already existing nanocrystal structure of another metal to enhance optical or electrochemical properties of the latter.

${ }^{2}$ Photo-blinking is defined as intermittent emission of fluorescent signals seen during continuous excitation of a fluorophore. 
Kurt, H., Yüce, M., Hussain, B., \& Budak, H. (2016). Dual-excitation upconverting nanoparticle and quantum dot aptasensor for multiplexed food pathogen detection. Biosensors and Bioelectronics, 81, 280-286.

Kushwaha, A., Takamura, Y., Nishigaki, K., \& Biyani, M. (2019). Competitive non-SELEX for the selective and rapid enrichment of DNA aptamers and its use in electrochemical aptasensor. Scientific Reports, 9(1), 6642.

Lai, Y.-T., \& DeStefano, J. J. (2012). Dna aptamers to human immunodeficiency virus reverse transcriptase selected by a primer-free selex method: Characterization and comparison with other aptamers. Nucleic Acid Therapeutics, 22(3), 162-176.

Lakhin, A. V., Tarantul, V. Z., \& Gening, L. V. (2013). Aptamers: Problems, solutions and prospects. Acta Naturae, 5(4), 34-43.

Law, J. W.-F., Ab Mutalib, N.-S., Chan, K.-G., \& Lee, L.-H. (2015). Rapid methods for the detection of foodborne bacterial pathogens: Principles, applications, advantages and limitations. Frontiers in Microbiology, 5 .

Leavesley, S. J., \& Rich, T. C. (2016). Overcoming limitations of FRET measurements. Cytometry Part A, $89(4), 325-327$.

Lee, B., Park, J., Ryu, M., Kim, S., Joo, M., Yeom, J.-H., Kim, S., Park, Y., Lee, K., \& Bae, J. (2017). Antimicrobial peptide-loaded gold nanoparticle-DNA aptamer conjugates as highly effective antibacterial therapeutics against Vibrio vulnificus. Scientific Reports, 7(1), 1-10.

Li, C.-H., Kuo, T.-R., Su, H.-J., Lai, W.-Y., Yang, P.-C., Chen, J.-S., Wang, D.-Y., Wu, Y.-C., \& Chen, C.-C. (2015). Fluorescence-guided probes of aptamer-targeted gold nanoparticles with computed tomography imaging accesses for in vivo tumor resection. Scientific Reports, 5.

Li, H., Ahmad, W., Rong, Y., Chen, Q., Zuo, M., Ouyang, Q., \& Guo, Z. (2020). Designing an aptamer based magnetic and upconversion nanoparticles conjugated fluorescence sensor for screening Escherichia coli in food. Food Control, 107, 106761.

Li, Y., Syed, J., \& Sugiyama, H. (2016). Rna-dna triplex formation by long noncoding rnas. Cell Chemical Biology, 23(11), 1325-1333.

Li, Yingfu. (2015). A quarter century of in vitro selection. Journal of Molecular Evolution, 81(5-6), 137-139.

Liao, Y.-J., Shiang, Y.-C., Huang, C.-C., \& Chang, H.-T. (2012). Molecularly imprinted aptamers of gold nanoparticles for the enzymatic inhibition and detection of thrombin. Langmuir, 28(24), 8944-8951.

Lichtman, J. W., \& Conchello, J.-A. (2005). Fluorescence microscopy. Nature Methods, 2(12), 910-919.

${ }^{1}$ Doping is defined as the method of introducing a metal ion called dopant (lanthanide or actinide in the text example) into the already existing nanocrystal structure of another metal to enhance optical or electrochemical properties of the latter.

${ }^{2}$ Photo-blinking is defined as intermittent emission of fluorescent signals seen during continuous excitation of a fluorophore. 
40 of 52

Lin, J. S., \& McNatty, K. P. (2009). Aptamer-based regionally protected pcr for protein detection. Clinical Chemistry, 55(9), 1686-1693.

Lipi, F., Chen, S., Chakravarthy, M., Rakesh, S., \& Veedu, R. N. (2016). In vitro evolution of chemicallymodified nucleic acid aptamers: Pros and cons, and comprehensive selection strategies. RNA Biology, 13(12), 1232-1245.

Liu, N., Zang, R., Yang, S.-T., \& Li, Y. (2014). Stem cell engineering in bioreactors for large-scale bioprocessing. Engineering in Life Sciences, 14(1), 4-15.

Liu, Q., Jin, C., Wang, Y., Fang, X., Zhang, X., Chen, Z., \& Tan, W. (2014). Aptamer-conjugated nanomaterials for specific cancer cell recognition and targeted cancer therapy. NPG Asia Materials, 6(4), e95-e95.

Lodish, H., Berk, A., Zipursky, S. L., Matsudaira, P., Baltimore, D., \& Darnell, J. (2000). Noncovalent bonds. Molecular Cell Biology. 4th Edition.

Lu, D., He, L., Zhang, G., Lv, A., Wang, R., Zhang, X., \& Tan, W. (2017). Aptamer-assembled nanomaterials for fluorescent sensing and imaging. Nanophotonics, 6(1), 109-121.

Lu, R.-M., Hwang, Y.-C., Liu, I.-J., Lee, C.-C., Tsai, H.-Z., Li, H.-J., \& Wu, H.-C. (2020). Development of therapeutic antibodies for the treatment of diseases. Journal of Biomedical Science, 27(1), 1.

MacDougall, C., Harpe, S. E., Powell, J. P., Johnson, C. K., Edmond, M. B., \& Polk, R. E. (2005). Pseudomonas aeruginosa, staphylococcus aureus, and fluoroquinolone use. Emerging Infectious Diseases, 11(8), 11971210.

Malik, M. T., O’Toole, M. G., Casson, L. K., Thomas, S. D., Bardi, G. T., Reyes-Reyes, E. M., Ng, C. K., Kang, K. A., \& Bates, P. J. (2015). AS1411-conjugated gold nanospheres and their potential for breast cancer therapy. Oncotarget, 6(26), 22270-22281.

Marks, L. (2012). The birth pangs of monoclonal antibody therapeutics: The failure and legacy of Centoxin. MAbs, 4(3), 403-412.

McKeague, M., McConnell, E. M., Cruz-Toledo, J., Bernard, E. D., Pach, A., Mastronardi, E., Zhang, X., Beking, M., Francis, T., Giamberardino, A., Cabecinha, A., Ruscito, A., Aranda-Rodriguez, R., Dumontier, M., \& DeRosa, M. C. (2015). Analysis of in vitro aptamer selection parameters. Journal of Molecular Evolution, 81(5-6), 150-161.

Md Alahi \& Subhas Mukhopadhyay. (2017). Detection methodologies for pathogen and toxins: A review. Sensors, 17(8), 1885.

Meyer, C., Hahn, U., \& Rentmeister, A. (2011). Cell-specific aptamers as emerging therapeutics. Journal of Nucleic Acids, 2011, 1-18.

${ }^{1}$ Doping is defined as the method of introducing a metal ion called dopant (lanthanide or actinide in the text example) into the already existing nanocrystal structure of another metal to enhance optical or electrochemical properties of the latter.

${ }^{2}$ Photo-blinking is defined as intermittent emission of fluorescent signals seen during continuous excitation of a fluorophore. 
41 of 52

Michaud, C. M. (2009). Global burden of infectious diseases. Encyclopedia of Microbiology, 444-454.

Mix, E., Goertsches, R., \& Zett, U. K. (2006). Immunoglobulins-Basic considerations. Journal of Neurology, 253(S5), v9-v17.

Morales, M. A., \& Halpern, J. M. (2018). Guide to selecting a biorecognition element for biosensors. Bioconjugate Chemistry, 29(10), 3231-3239.

Mori, T., \& Katayama, Y. (2019). Signal amplification in flow cytometry for cell surface antigen analysis. Journal of Biochemistry, 166(3), 205-212.

Musumeci, D., Platella, C., Riccardi, C., Moccia, F., \& Montesarchio, D. (2017). Fluorescence sensing using dna aptamers in cancer research and clinical diagnostics. Cancers, 9(12), 174.

National Research Council (US) Committee on Methods of Producing Monoclonal Antibodies. (1999). Monoclonal antibody production. National Academies Press (US).

Neubacher, S., \& Hennig, S. (2019). Rna structure and cellular applications of fluorescent light-up aptamers. Angewandte Chemie, 131(5), 1278-1291.

Ni, S., Yao, H., Wang, L., Lu, J., Jiang, F., Lu, A., \& Zhang, G. (2017). Chemical modifications of nucleic acid aptamers for therapeutic purposes. International Journal of Molecular Sciences, 18(8), 1683.

Ni, S., Zhuo, Z., Pan, Y., Yu, Y., Li, F., Liu, J., Wang, L., Wu, X., Li, D., Wan, Y., Zhang, L., Yang, Z., Zhang, B.-T., Lu, A., \& Zhang, G. (2020). Recent progress in aptamer discoveries and modifications for therapeutic applications. ACS Applied Materials \& Interfaces, acsami.0c05750.

Nimjee, S. M., Rusconi, C. P., \& Sullenger, B. A. (2005). Aptamers: An emerging class of therapeutics. Annual Review of Medicine, 56(1), 555-583.

Noh, J. Y., Yoon, S.-W., Kim, Y., Lo, T. V., Ahn, M.-J., Jung, M.-C., Le, T. B., Na, W., Song, D., Le, V. P., Haam, S., Jeong, D. G., \& Kim, H. K. (2019). Pipetting-based immunoassay for point-of-care testing: Application for detection of the influenza A virus. Scientific Reports, 9(1), 16661.

Ohuchi, S. (2012). Cell-selex technology. BioResearch Open Access, 1(6), 265-272.

Orava, E. W., Cicmil, N., \& Gariépy, J. (2010). Delivering cargoes into cancer cells using DNA aptamers targeting internalized surface portals. Biochimica Et Biophysica Acta, 1798(12), 2190-2200.

Paige, J. S., Nguyen-Duc, T., Song, W., \& Jaffrey, S. R. (2012). Fluorescence imaging of cellular metabolites with RNA. Science (New York, N.Y.), 335(6073), 1194.

Palchetti, I., \& Mascini, M. (2008). Nucleic acid biosensors for environmental pollution monitoring. The Analyst, 133(7), 846-854.

${ }^{1}$ Doping is defined as the method of introducing a metal ion called dopant (lanthanide or actinide in the text example) into the already existing nanocrystal structure of another metal to enhance optical or electrochemical properties of the latter.

${ }^{2}$ Photo-blinking is defined as intermittent emission of fluorescent signals seen during continuous excitation of a fluorophore. 
Pan, W., \& Clawson, G. (2009). The shorter the better: Reducing fixed primer regions of oligonucleotide libraries for aptamer selection. Molecules, 14(4), 1353-1369.

Pang, X., Cui, C., Wan, S., Jiang, Y., Zhang, L., Xia, L., Li, L., Li, X., \& Tan, W. (2018). Bioapplications of cellselex-generated aptamers in cancer diagnostics, therapeutics, theranostics and biomarker discovery: A comprehensive review. Cancers, 10(2).

Paniel \& Noguer. (2019). Detection of salmonella in food matrices, from conventional methods to recent aptamer-sensing technologies. Foods, 8(9), 371.

Pastor, F., Berraondo, P., Etxeberria, I., Frederick, J., Sahin, U., Gilboa, E., \& Melero, I. (2018). An RNA toolbox for cancer immunotherapy. Nature Reviews Drug Discovery, 17(10), 751-767.

Pereira, D. A., \& Williams, J. A. (2007). Origin and evolution of high throughput screening. British Journal of Pharmacology, 152(1), 53.

Peselis, A., \& Serganov, A. (2014). Structure and function of pseudoknots involved in gene expression control: Structure and function of pseudoknots. Wiley Interdisciplinary Reviews: RNA, 5(6), 803-822.

Phillips, J. A., Xu, Y., Xia, Z., Fan, Z. H., \& Tan, W. (2009). Enrichment of cancer cells using aptamers immobilized on a microfluidic channel. Analytical Chemistry, 81(3), 1033-1039.

Pisanic II, T. R., Zhang, Y., \& Wang, T. H. (2014). Quantum dots in diagnostics and detection: Principles and paradigms. The Analyst, 139(12), 2968-2981.

Pobanz, K., \& Lupták, A. (2016). Improving the odds: Influence of starting pools on in vitro selection outcomes. Methods (San Diego, Calif.), 106, 14.

Pramanik, A., Fan, Z., Chavva, S. R., Sinha, S. S., \& Ray, P. C. (2014). Highly efficient and excitation tunable two-photon luminescence platform for targeted multi-color mdrb imaging using graphene oxide. Scientific Reports, 4 .

Prusty, D. K., Adam, V., Zadegan, R. M., Irsen, S., \& Famulok, M. (2018). Supramolecular aptamer nanoconstructs for receptor-mediated targeting and light-triggered release of chemotherapeutics into cancer cells. Nature Communications, 9.

Rahimi, F. (2018). Aptamers selected for recognizing amyloid $\beta$-protein-A case for cautious optimism. International Journal of Molecular Sciences, 19(3), 668.

Rajapaksha, P., Elbourne, A., Gangadoo, S., Brown, R., Cozzolino, D., \& Chapman, J. (2019). A review of methods for the detection of pathogenic microorganisms. The Analyst, 144(2), 396-411.

${ }^{1}$ Doping is defined as the method of introducing a metal ion called dopant (lanthanide or actinide in the text example) into the already existing nanocrystal structure of another metal to enhance optical or electrochemical properties of the latter.

${ }^{2}$ Photo-blinking is defined as intermittent emission of fluorescent signals seen during continuous excitation of a fluorophore. 
Ravindranath, S. P., Wang, Y., \& Irudayaraj, J. (2011). SERS driven cross-platform based multiplex pathogen detection. Sensors and Actuators B: Chemical, 152(2), 183-190.

Rietveld, K., Van Poelgeest, R., Pleij, C. W. A., Van Boom, J. H., \& Bosch, L. (1982). The tRNA-Uke structure at the $3^{\prime}$ terminus of turnip yellow mosaic virus RNA. Differences and similarities with canonical tRNA. Nucleic Acids Research, 10(6), 1929-1946.

Roberts, R., \& Crothers, D. (1992). Stability and properties of double and triple helices: Dramatic effects of RNA or DNA backbone composition. Science, 258(5087), 1463-1466.

Robertson, D. L., \& Joyce, G. F. (1990). Selection in vitro of an RNA enzyme that specifically cleaves singlestranded DNA. Nature, 344(6265), 467-468.

Ruiz Ciancio, D., Vargas, M., Thiel, W., Bruno, M., Giangrande, P., \& Mestre, M. (2018). Aptamers as diagnostic tools in cancer. Pharmaceuticals, 11(3), 86.

Sakamoto, S., Putalun, W., Vimolmangkang, S., Phoolcharoen, W., Shoyama, Y., Tanaka, H., \& Morimoto, S. (2018). Enzyme-linked immunosorbent assay for the quantitative/qualitative analysis of plant secondary metabolites. Journal of Natural Medicines, 72(1), 32-42.

Schasfoort, R. B. M. (2017). Chapter 1. Introduction to surface plasmon resonance. In R. B. M. Schasfoort (Ed.), Handbook of Surface Plasmon Resonance (2nd ed., pp. 1-26). Royal Society of Chemistry.

Schuster, J., Mahler, H.-C., Koulov, A., Joerg, S., Racher, A., Huwyler, J., Detampel, P., \& Mathaes, R. (2020). Tracking the physical stability of fluorescent-labeled $\mathrm{mAbs}$ under physiologic in vitro conditions in human serum and PBS. European Journal of Pharmaceutics and Biopharmaceutics, 152, 193-201.

Sefah, K., Shangguan, D., Xiong, X., O'Donoghue, M. B., \& Tan, W. (2010). Development of dna aptamers using cell-selex. Nature Protocols, 5(6), 1169-1185.

Seo, Y.-J., Chen, S., Nilsen-Hamilton, M., \& Levine, H. A. (2010). A mathematical analysis of multiple-target selex. Bulletin of Mathematical Biology, 72(7), 1623-1665.

Shangguan, D., Li, Y., Tang, Z., Cao, Z. C., Chen, H. W., Mallikaratchy, P., Sefah, K., Yang, C. J., \& Tan, W. (2006). Aptamers evolved from live cells as effective molecular probes for cancer study. Proceedings of the National Academy of Sciences, 103(32), 11838-11843.

Shi, H., He, X., Wang, K., Wu, X., Ye, X., Guo, Q., Tan, W., Qing, Z., Yang, X., \& Zhou, B. (2011). Activatable aptamer probe for contrast-enhanced in vivo cancer imaging based on cell membrane protein-triggered conformation alteration. Proceedings of the National Academy of Sciences, 108(10), 3900-3905.

${ }^{1}$ Doping is defined as the method of introducing a metal ion called dopant (lanthanide or actinide in the text example) into the already existing nanocrystal structure of another metal to enhance optical or electrochemical properties of the latter.

${ }^{2}$ Photo-blinking is defined as intermittent emission of fluorescent signals seen during continuous excitation of a fluorophore. 
44 of 52

Sola, M., Menon, A. P., Moreno, B., Meraviglia-Crivelli, D., Soldevilla, M. M., Cartón-García, F., \& Pastor, F. (2020). Aptamers against live targets: Is in vivo selex finally coming to the edge? Molecular Therapy Nucleic Acids, 21, 192-204.

Song, K.-M., Lee, S., \& Ban, C. (2012). Aptamers and their biological applications. Sensors, 12(1), 612-631.

Song, M. Y., Nguyen, D., Hong, S. W., \& Kim, B. C. (2017). Broadly reactive aptamers targeting bacteria belonging to different genera using a sequential toggle cell-SELEX. Scientific Reports, 7.

Song, R. L. S., Wenjiao, \& Jaffrey, S. R. (2014). Using Spinach-based sensors for fluorescence imaging of intracellular metabolites and proteins in living bacteria. Nature Protocols, 9(1), 146-155.

Song, S., Wang, L., Li, J., Fan, C., \& Zhao, J. (2008). Aptamer-based biosensors. TrAC Trends in Analytical Chemistry, 27(2), 108-117.

Soundy, J., \& Day, D. (2017). Selection of DNA aptamers specific for live Pseudomonas aeruginosa. PLOS ONE, 12(9), e0185385.

Spill, F., Weinstein, Z. B., Irani Shemirani, A., Ho, N., Desai, D., \& Zaman, M. H. (2016). Controlling uncertainty in aptamer selection. Proceedings of the National Academy of Sciences, 113(43), 12076-12081.

Stoltenburg, R., Reinemann, C., \& Strehlitz, B. (2007). SELEX-A (R)evolutionary method to generate highaffinity nucleic acid ligands. Biomolecular Engineering, 24(4), 381-403.

Subramanian, N., Kanwar, J. R., Kanwar, R. K., Sreemanthula, J., Biswas, J., Khetan, V., \& Krishnakumar, S. (2015). Epcam aptamer-sirna chimera targets and regress epithelial cancer. PLOS ONE, 10(7), e0132407.

Sullenger, B. A., Gallardo, H. F., Ungers, G. E., \& Gilboa, E. (1990). Overexpression of TAR sequences renders cells resistant to human immunodeficiency virus replication. Cell, 63(3), 601-608.

Sun, F., Galas, D., \& Waterman, M. S. (1996). A mathematical analysis of in vitro molecular selection amplification. Journal of Molecular Biology, 258(4), 650-660.

Sun, P., Lu, X., Fan, Q., Zhang, Z., Song, W., Li, B., Huang, L., Peng, J., \& Huang, W. (2011). Water-soluble iridium(Iii)-containing conjugated polyelectrolytes with weakened energy transfer properties for multicolor protein sensing applications. Macromolecules, 44(22), 8763-8770.

Svoboda, P., \& Cara, A. Di. (2006). Hairpin RNA: A secondary structure of primary importance. Cellular and Molecular Life Sciences, 63(7-8), 901-908.

Szabat, M., \& Kierzek, R. (2017). Parallel-stranded DNA and RNA duplexes-Structural features and potential applications. The FEBS Journal, 284(23), 3986-3998.

${ }^{1}$ Doping is defined as the method of introducing a metal ion called dopant (lanthanide or actinide in the text example) into the already existing nanocrystal structure of another metal to enhance optical or electrochemical properties of the latter.

${ }^{2}$ Photo-blinking is defined as intermittent emission of fluorescent signals seen during continuous excitation of a fluorophore. 
45 of 52

Taghdisi, S. M., Abnous, K., Mosaffa, F., \& Behravan, J. (2010). Targeted delivery of daunorubicin to T-cell acute lymphoblastic leukemia by aptamer. Journal of Drug Targeting, 18(4), 277-281.

Tan, J., Yang, N., Zhong, L., Tan, J., Hu, Z., Zhao, Q., Gong, W., Zhang, Z., Zheng, R., Lai, Z., Li, Y., Zhou, C., Zhang, G., Zheng, D., Zhang, Y., Wu, S., Jiang, X., Zhong, J., Huang, Y., ... Zhao, Y. (2017). A new theranostic system based on endoglin aptamer conjugated fluorescent silica nanoparticles. Theranostics, 7(19), 4862-4876.

Tang, J., Huang, N., Zhang, X., Zhou, T., Tan, Y., Pi, J., Pi, L., Cheng, S., Zheng, H., \& Cheng, Y. (2017). Aptamer-conjugated PEGylated quantum dots targeting epidermal growth factor receptor variant III for fluorescence imaging of glioma. International Journal of Nanomedicine, 12, 3899-3911.

Tang, Z., Shangguan, D., Wang, K., Shi, H., Sefah, K., Mallikratchy, P., Chen, H. W., Li, Y., \& Tan, W. (2007). Selection of aptamers for molecular recognition and characterization of cancer cells. Analytical Chemistry, 79(13), 4900-4907.

Thiel, W. H., Bair, T., Wyatt Thiel, K., Dassie, J. P., Rockey, W. M., Howell, C. A., Liu, X. Y., Dupuy, A. J., Huang, L., Owczarzy, R., Behlke, M. A., McNamara, J. O., \& Giangrande, P. H. (2011). Nucleotide bias observed with a short selex rna aptamer library. Nucleic Acid Therapeutics, 21(4), 253-263.

Tok, J. B.-H. (2000). RNA aptamers that specifically bind to a $16 S$ ribosomal RNA decoding region construct. Nucleic Acids Research, 28(15), 2902-2910.

Tolle, F., Wilke, J., Wengel, J., \& Mayer, G. (2014). By-product formation in repetitive pcr amplification of dna libraries during selex. PLoS ONE, 9(12), e114693.

Tuerk, C., \& Gold, L. (1990). Systematic evolution of ligands by exponential enrichment: RNA ligands to bacteriophage T4 DNA polymerase. Science, 249(4968), 505-510.

Using aptamers to control enzyme activity: Hot Start Taq and beyond. NEB. Retrieved September 16, 2020, from https://www.neb.com/tools-and-resources/feature-articles/using-aptamers-to-control-enzymeactivities-hot-start-taq-and-beyond

van Gijtenbeek, L. A., \& Kok, J. (2017). Illuminating messengers: An update and outlook on rna visualization in bacteria. Frontiers in Microbiology, 8.

Vant-Hull, B., Payano-Baez, A., Davis, R. H., \& Gold, L. (1998). The mathematics of SELEX against complex targets. Journal of Molecular Biology, 278(3), 579-597.

Varani, G. (1995). Exceptionally stable nucleic acid hairpins. Annual Review of Biophysics and Biomolecular Structure, 24(1), 379-404.

${ }^{1}$ Doping is defined as the method of introducing a metal ion called dopant (lanthanide or actinide in the text example) into the already existing nanocrystal structure of another metal to enhance optical or electrochemical properties of the latter.

2 Photo-blinking is defined as intermittent emission of fluorescent signals seen during continuous excitation of a fluorophore. 
46 of 52

Vater, A., \& Klussmann, S. (2015). Turning mirror-image oligonucleotides into drugs: The evolution of Spiegelmer ${ }^{\circledR}$ therapeutics. Drug Discovery Today, 20(1), 147-155.

Vidic, M., Smuc, T., Janez, N., Blank, M., Accetto, T., Mavri, J., Nascimento, I. C., Nery, A. A., Ulrich, H., \& Lah, T. T. (2018). In silico selection approach to develop dna aptamers for a stem-like cell subpopulation of non-small lung cancer adenocarcinoma cell line a549. Radiology and Oncology, 52(2), 152-159.

Vivekananda, J., Salgado, C., \& Millenbaugh, N. J. (2014). DNA aptamers as a novel approach to neutralize Staphylococcus aureus $\alpha$-toxin. Biochemical and Biophysical Research Communications, 444(3), 433-438.

Vorobyeva, M., Davydova, A., Vorobjev, P., Pyshnyi, D., \& Venyaminova, A. (2018). Key aspects of nucleic acid library design for in vitro selection. International Journal of Molecular Sciences, 19(2), 470.

Vu CQ, Tantirungrotechai Y, and Soontornworajit B. Truncation of PDGF-BB Aptamer by Secondary Structural Analysis and Immunoassay (2016) . International Journal of Pharma Medicine and Biological Sciences Vol. 5, No. 1, p. 86, 10.18178/ijpmbs.5.1.86-90

Wade, N. (1980). Inventor of hybridoma technology failed to file for patent. Science, 208(4445), 693-693.

Wang, B. (2020). A new design for the fixed primer regions in an oligonucleotide library for selex aptamer screening. Frontiers in Chemistry, 8, 475.

Wang, J., Rudzinski, J. F., Gong, Q., Soh, H. T., \& Atzberger, P. J. (2012). Influence of target concentration and background binding on in vitro selection of affinity reagents. PLoS ONE, 7(8), e43940.

Wang, K.-Y., Zeng, Y.-L., Yang, X.-Y., Li, W.-B., \& Lan, X.-P. (2011). Utility of aptamer-fluorescence in situ hybridization for rapid detection of Pseudomonas aeruginosa. European Journal of Clinical Microbiology \& Infectious Diseases, 30(2), 273-278.

Wang, M., Abbineni, G., Clevenger, A., Mao, C., \& Xu, S. (2011). Upconversion nanoparticles: Synthesis, surface modification and biological applications. Nanomedicine: Nanotechnology, Biology and Medicine, $7(6), 710-729$.

Wang, Q.Y., \& Kang, Y.J. (2016). Bioprobes based on aptamer and silica fluorescent nanoparticles for bacteria salmonella typhimurium detection. Nanoscale Research Letters, 11.

Wang, R., Xu, Y., Zhang, T., \& Jiang, Y. (2015). Rapid and sensitive detection of Salmonella typhimurium using aptamer-conjugated carbon dots as fluorescence probe. Analytical Methods, 7(5), 1701-1706.

Wang, T., Chen, C., Larcher, L. M., Barrero, R. A., \& Veedu, R. N. (2019). Three decades of nucleic acid aptamer technologies: Lessons learned, progress and opportunities on aptamer development. Biotechnology Advances, 37(1), 28-50.

${ }^{1}$ Doping is defined as the method of introducing a metal ion called dopant (lanthanide or actinide in the text example) into the already existing nanocrystal structure of another metal to enhance optical or electrochemical properties of the latter.

${ }^{2}$ Photo-blinking is defined as intermittent emission of fluorescent signals seen during continuous excitation of a fluorophore. 
Wang, T., Gantier, M. P., Xiang, D., Bean, A. G., Bruce, M., Zhou, S.-F., Khasraw, M., Ward, A., Wang, L., Wei, M. Q., AlShamaileh, H., Chen, L., She, X., Lin, J., Kong, L., Shigdar, S., \& Duan, W. (2015). Epcam aptamer-mediated survivin silencing sensitized cancer stem cells to doxorubicin in a breast cancer model. Theranostics, 5(12), 1456-1472.

Widmann, J., Giulio, M. D., Yarus, M., \& Knight, R. (2005). Trna creation by hairpin duplication. Journal of Molecular Evolution, 61(4), 524-530.

Wu, C., Liu, J., Zhang, P., Li, J., Ji, H., Yang, X., \& Wang, K. (2015). A recognition-before-labeling strategy for sensitive detection of lung cancer cells with a quantum dot-aptamer complex. The Analyst, 140(17), 6100-6107.

Wu, X., Chen, J., Wu, M., \& Zhao, J. X. (2015). Aptamers: Active targeting ligands for cancer diagnosis and therapy. Theranostics, 5(4), 322-344.

Xing, H., Tang, L., Yang, X., Hwang, K., Wang, W., Yin, Q., Wong, N. Y., Dobrucki, L. W., Yasui, N., Katzenellenbogen, J. A., Helferich, W. G., Cheng, J., \& Lu, Y. (2013). Selective delivery of an anticancer drug with aptamer-functionalized liposomes to breast cancer cells in vitro and in vivo. Journal of Materials Chemistry B, 1(39), 5288.

Yang, X., Li, N., \& Gorenstein, D. G. (2011). Strategies for the discovery of therapeutic Aptamers. Expert Opinion on Drug Discovery, 6(1), 75-87.

Yoo, S. M., Kim, D.-K., \& Lee, S. Y. (2015). Aptamer-functionalized localized surface plasmon resonance sensor for the multiplexed detection of different bacterial species. Talanta, 132, 112-117.

Yoon, S., \& Rossi, J. J. (2018). Aptamers: Uptake mechanisms and intracellular applications. Advanced Drug Delivery Reviews, 134, 22-35.

Yoon, S., \& Rossi, J. J. (2018). Targeted molecular imaging using aptamers in cancer. Pharmaceuticals, 11(3).

Zelada-Guillén, G. A., Riu, J., Düzgün, A., \& Rius, F. X. (2009). Immediate detection of living bacteria at ultralow concentrations using a carbon nanotube based potentiometric aptasensor. Angewandte Chemie International Edition, 48(40), 7334-7337.

Zeng, Z., Tung, C.-H., \& Zu, Y. (2014). A cancer cell-activatable aptamer-reporter system for one-step assay of circulating tumor cells. Molecular Therapy - Nucleic Acids, 3, e184.

Zhang, C., Fu, H., Yang, Y., Zhou, E., Tan, Z., You, H., \& Zhang, X. (2019). The mechanical properties of rnadna hybrid duplex stretched by magnetic tweezers. Biophysical Journal, 116(2), 196-204.

Zhang, Y., Hong, H., \& Cai, W. (2011). Tumor-targeted drug delivery with aptamers. Current Medicinal Chemistry, 18(27), 4185-4194.

${ }^{1}$ Doping is defined as the method of introducing a metal ion called dopant (lanthanide or actinide in the text example) into the already existing nanocrystal structure of another metal to enhance optical or electrochemical properties of the latter.

${ }^{2}$ Photo-blinking is defined as intermittent emission of fluorescent signals seen during continuous excitation of a fluorophore. 
48 of 52

Zhang, Z., Oni, O., \& Liu, J. (2017). New insights into a classic aptamer: Binding sites, cooperativity and more sensitive adenosine detection. Nucleic Acids Research, 45(13), 7593-7601.

Zhao, X., Lu, C., Yang, S., \& Zhang, J. (2020). Bioconjugation of aptamer to fluorescent trimethyl chitosan nanoparticles for bacterial detection. Materials Letters, 264, 127330.

Zheng, D., Zou, R., \& Lou, X. (2012). Label-free fluorescent detection of ions, proteins, and small molecules using structure-switching aptamers, sybr gold, and exonuclease i. Analytical Chemistry, 84(8), 3554-3560.

Zhou, J., \& Rossi, J. (2017). Aptamers as targeted therapeutics: Current potential and challenges. Nature Reviews Drug Discovery, 16(3), 181-202.

Zhou, J., Soontornworajit, B., Martin, J., Sullenger, B. A., Gilboa, E., \& Wang, Y. (2009). A hybrid dna aptamer-dendrimer nanomaterial for targeted cell labeling: A hybrid dna aptamer-dendrimer nanomaterial for targeted cell labeling. Macromolecular Bioscience, 9(9), 831-835.

Zhu, G., Niu, G., \& Chen, X. (2015). Aptamer-drug conjugates. Bioconjugate Chemistry, 26(11), 2186-2197.

Zhu, Q., Liu, G., \& Kai, M. (2015). Dna aptamers in the diagnosis and treatment of human diseases. Molecules, 20(12), 20979-20997.

Zhuo, Z., Yu, Y., Wang, M., Li, J., Zhang, Z., Liu, J., Wu, X., Lu, A., Zhang, G., \& Zhang, B. (2017). Recent advances in selex technology and aptamer applications in biomedicine. International Journal of Molecular Sciences, 18(10), 2142.

Zrazhevskiy, P., Sena, M., \& Gao, X. (2010). Designing multifunctional quantum dots for bioimaging, detection, and drug delivery. Chemical Society Reviews, 39(11), 4326.

${ }^{1}$ Doping is defined as the method of introducing a metal ion called dopant (lanthanide or actinide in the text example) into the already existing nanocrystal structure of another metal to enhance optical or electrochemical properties of the latter.

${ }^{2}$ Photo-blinking is defined as intermittent emission of fluorescent signals seen during continuous excitation of a fluorophore. 
Table 1.1 Examples of fluorescent aptasensor designs used as diagnostic and therapeutic agents targeting cancer or bacterial pathogens

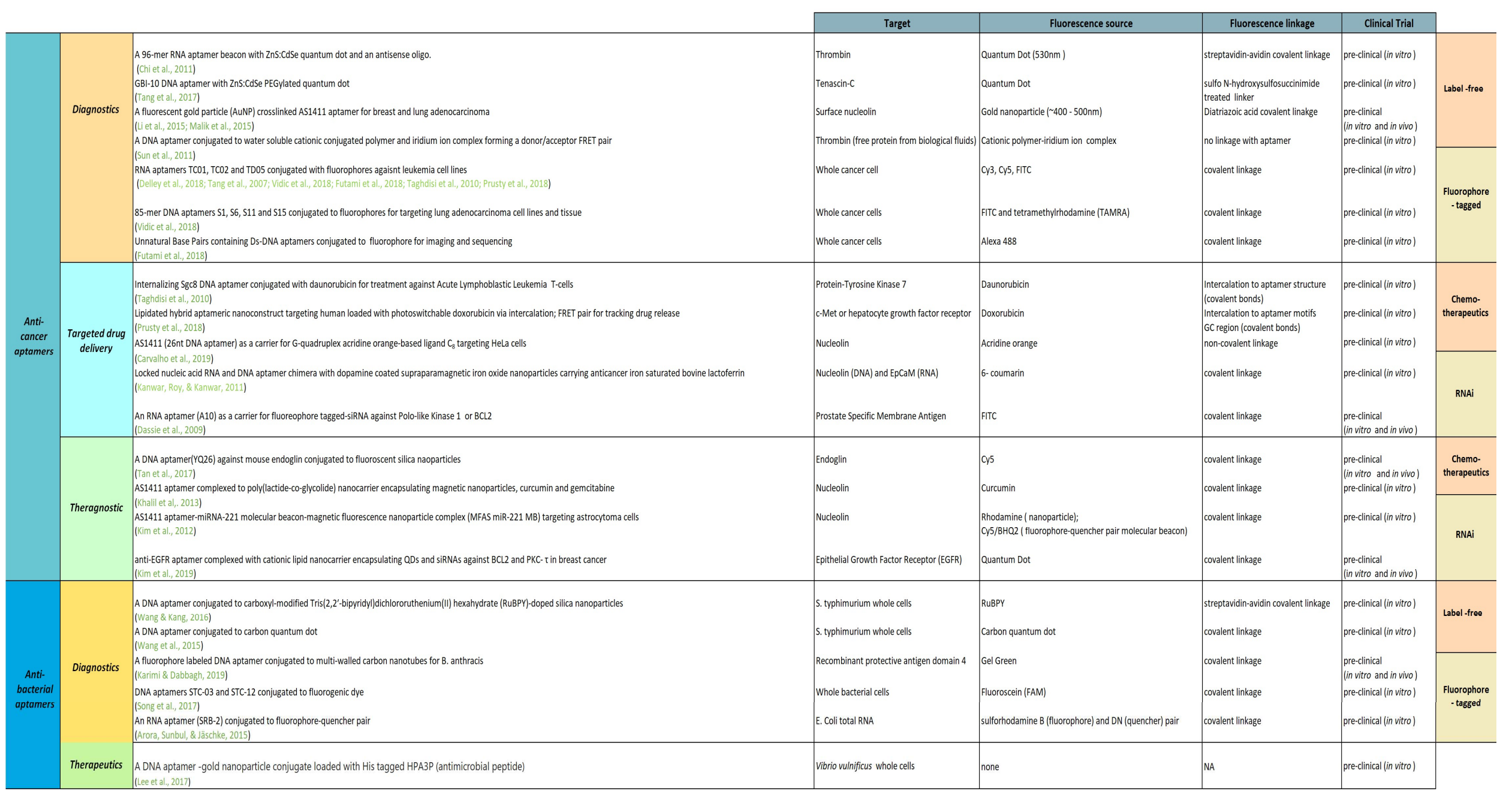




\section{Abbreviations}

DNA- Deoxyribonucleic acid

RNA- Ribonucleic acid

SELEX- Systematic evolution of ligands by exponential enrichment

PCR- Polymerase Chain Reaction

RT-PCR- Reverse Transcription Polymerase Chain Reaction

cDNA- complementary DNA

PAGE- Polyacrylamide gel electrophoresis

PEG- Polyethylene glycol

IgM- Immunoglobulin M

SiRNA- Short interfering RNA

miRNA- micro RNA

AsiC- Aptamer siRNA complex

AmiC- Aptamer miRNA complex

MUC1- Mucin 1, a polymorphic epithelial mucin

CD38- cluster of differentiation 38; also known as cyclic ADP ribose hydrolase

LAMP- Loop-mediated isothermal amplification

NASBA- Nucleic acid sequence-based amplification

FRET- Förster resonance energy transfer

SPR- Surface Plasma Resonance

NMR- Nuclear Magnetic Resonance

ELISA- Enzyme-Linked Immunosorbent Assay

LFA- Lateral Flow Assay

AuNP- Gold nanoparticle 
QD- Quantum Dot

SERS- Surface-enhanced Raman Spectroscopy

CFU- Colony Forming Units

FISH- Fluorescence in-situ hybridization

UCNP- Upconverting nanoparticle

eGFP- Enhanced Green Fluorescent Protein

ADP- Adenosine diphosphate

FLAP- Florescence light-up aptamer

SAM- S-adenosylmethionine

ARP-PCR-Aptamer-based regionally protected PCR

RAPID- RNA aptamer in the droplet

MDR- Multi-drug resistant

EGFR- Epidermal Growth Factor Receptor

EpCAM- Epithelial cell adhesion molecule

Cy5-Cyanine 5, a synthetic red fluorescent dye

CTC- Circulating tumor cells

IncRNA- Long non-coding RNA

PMCA- Prostate-specific membrane antigen

T-ALL- T-cell acute lymphoblastic leukemia

\footnotetext{
${ }^{1}$ Doping is defined as the method of introducing a metal ion called dopant (lanthanide or actinide in the text example) into the already existing nanocrystal structure of another metal to enhance optical or electrochemical properties of the latter.

${ }^{2}$ Photo-blinking is defined as intermittent emission of fluorescent signals seen during continuous excitation of a fluorophore.
} 
MTX- Methotrexate

CD117- Cluster of Differentiation 117, a receptor tyrosine kinase encoded by c-KIT gene. EpCAM

NHS- N-hydroxysuccinimide

AML- Acute Myeloid Leukemia

PLK1- Polo-like kinase

TRAIL- TNF- alpha Related Apoptosis Inducing Ligand

FDA- Federal Drug Administration

CTLA4- Cytotoxic T-lymphocyte-associated protein 4 or CD152; an immune checkpoint

PD1- Programmed cell death protein1

MMAE- Monomethyl auristatin E

MMAF- Monomethyl auristatin F

\footnotetext{
${ }^{1}$ Doping is defined as the method of introducing a metal ion called dopant (lanthanide or actinide in the text example) into the already existing nanocrystal structure of another metal to enhance optical or electrochemical properties of the latter.

${ }^{2}$ Photo-blinking is defined as intermittent emission of fluorescent signals seen during continuous excitation of a fluorophore.
} 